\title{
Doubly-charged Higgs and vacuum stability in left-right supersymmetry
}

\author{
Lorenzo Basso, ${ }^{a}$ Benjamin Fuks, ${ }^{a}$ Manuel E. Krauss ${ }^{b}$ and Werner Porod ${ }^{b}$ \\ ${ }^{a}$ Institut Pluridisciplinaire Hubert Curien, Département Recherches Subatomiques, \\ Université de Strasbourg, CNRS-IN2P3, \\ 23 rue du Loess, F-67037 Strasbourg, France \\ ${ }^{b}$ Institut für Theoretische Physik und Astronomie, Universität Würzburg, \\ Emil-Hilb-Weg 22, 97074 Würzburg, Germany \\ E-mail: lorenzo.basso@iphc.cnrs.fr, benjamin.fuks@iphc.cnrs.fr, \\ manuel.krauss@physik.uni-wuerzburg.de, porod@physik.uni-wuerzburg.de
}

ABSTRACT: We present an analysis of supersymmetric left-right symmetric models with Higgs fields lying in the adjoint representation of $\mathrm{SU}(2)_{R}$. These models feature a doublycharged Higgs boson which gets its mass only at the loop level. We present, for the first time, a complete one-loop calculation of this mass and show that contributions that have been neglected so far can shift it by a few hundreds of $\mathrm{GeV}$. We then combine this observation with LHC bounds deduced from extra charged gauge boson and doubly-charged Higgs boson searches. In particular, we point out that existing limits get substantially modified by the presence of singly-charged Higgs bosons that are also predicted in these models. In addition, we address constraints stemming from vacuum stability considerations and show how the considered class of models could be ruled out at the next LHC run in the absence of any signal.

KEYWORDS: Supersymmetry Phenomenology

ARXiv EPrint: 1503.08211 


\section{Contents}

1 Introduction 1

2 The Model 3

3 The doubly-charged Higgs mass 4

3.1 Tree-level results 5

$\begin{array}{ll}3.2 & \text { Results at the one-loop level }\end{array}$

4 LHC bounds $\quad 11$

5 Analysis of the vacuum stability 14

6 Conclusion 20

\section{Introduction}

The Large Hadron Collider (LHC) at CERN has been built to explore the TeV energy scale and pin down the mechanism responsible for the breaking of the electroweak symmetry. With this respect, the discovery of a Higgs boson consistent with the Standard Model of particle physics $[1,2]$ can be seen as a first success of the LHC physics programme. This also consists of the first observation of a particle intrinsically unstable with respect to quantum corrections. One can therefore expect either an unnatural fine-tuning or a stabilization arising from physics beyond the Standard Model that would emerge at a scale reachable at present and future colliders. Although we do not know which theory could be the most suitable, a lot of efforts have been put, during the last decades, in the study of a plethora of new physics models. The Minimal Supersymmetric Standard Model [3, 4] (MSSM) is one of the most popular of these and it provides, additionally to curing the infamous hierarchy problem, a solution for other conceptual issues plaguing the Standard Model.

There is currently no evidence for supersymmetry and limits on the superpartners of the Standard Model particles are hence pushed to higher and higher scales. Most results have however been derived either in the MSSM or within simplified models inspired by the latter. There are nevertheless large varieties of alternative supersymmetric realizations that could evade all current bounds and deserve to be investigated. For instance, the MSSM inherits some of the flaws of the Standard Model and extending the model gauge symmetry could provide a mechanism yielding the generation of the neutrino masses and an explanation for the strong $C P$ problem.

We consider in this work supersymmetric theories that exhibit a left-right symmetry [58] and that are based on an $\mathrm{SU}(3)_{c} \times \mathrm{SU}(2)_{L} \times \mathrm{SU}(2)_{R} \times \mathrm{U}(1)_{B-L}$ gauge group. They provide a solution for the hierarchy problem, explain the smallness of the neutrino masses, can include a dark matter candidate, and they potentially solve the strong $C P$ problem 
because of parity invariance at the fundamental level. Moreover, minimal supersymmetric setups like constrained versions of the MSSM cannot usually predict, at the tree level, a neutral Higgs boson mass compatible with the observations and require large higher-order contributions. This issue can be alleviated by extending the Higgs sector, like in left-right supersymmetric models where the spontaneous breaking of the left-right symmetry all the way down to the U(1) electromagnetic group necessitates several Higgs fields [9-11].

Several left-right supersymmetric setups have been studied in the past, with different Higgs sectors and symmetry breaking details. We choose to focus on a class of minimal models featuring two $\mathrm{SU}(2)_{R}$ Higgs triplets that are responsible for the breaking of $\mathrm{SU}(2)_{R} \times$ $\mathrm{U}(1)_{B-L}$ to the electroweak group, two extra $\mathrm{SU}(2)_{L}$ Higgs triplets that allow for parity invariance at higher scales and two $\mathrm{SU}(2)_{L} \times \mathrm{SU}(2)_{R}$ bidoublets yielding both the breaking of the electroweak symmetry down to electromagnetism and the generation of acceptable fermion masses and mixings [12-14]. In addition, we include an extra singlet field to achieve a successful electroweak symmetry breaking in the supersymmetric limit [8]. With such a choice for the Higgs sector, the interactions of the lepton supermultiplets with the triplets are sufficient for generating neutrino masses through a see-saw mechanism [15], and the presence of the singlet solves the so-called $\mu$-problem of the MSSM as all bilinear terms of the superpotential can be generated dynamically. These models are currently probed at the LHC through traditional supersymmetry $[16,17]$ and extra gauge boson searches $[18,19]$, as well as analyses dedicated to the doubly-charged (Higgs and Higgsino) states induced by the $\mathrm{SU}(2)_{L}$ and $\mathrm{SU}(2)_{R}$ triplets $[18,19]$. The observation of such doubly-charged particles would indeed provide valuable information about the symmetry breaking pattern of the underlying theory.

The minimization of the scalar potential of the model is known to lead to a tree-level solution in which either $R$-parity invariance $[12,13]$ or electric charge conservation [14] is lost. Including one-loop heavy Majorana neutrino contributions however allows for satisfactory solutions to the minimization equations $[8,20]$. In this case, the right-handed sneutrino fields are prevented from getting non-vanishing vacuum expectation values so that $R$-parity is conserved and no dangerous lepton number violating operators appear in the superpotential. Additionally, these radiative corrections also protect the doubly-charged Higgs bosons from being tachyonic and breaking electromagnetism. This has been demonstrated both in pioneering calculations relying on the Coleman-Weinberg effective potential [8] and in a more recent calculation using a diagrammatic approach in the gaugeless limit where all contributions proportional to the gauge couplings are neglected [20].

In this work, we start with a brief description of the considered left-right supersymmetric models (section 2) and then show, in section 3, that the previously calculated corrections to the doubly-charged Higgs mass are not sufficient to render it compatible with the current experimental bounds [21]. We subsequently evaluate the complete oneloop result and demonstrate that the doubly-charged Higgs mass can be increased well above the present limits. Other recent LHC constraints are investigated in section 4 and we further study the minimum of the scalar potential at the one-loop level in section 5, analyzing the requirements for ensuring this minimum to be global. Our conclusions about the phenomenological viability of the model are given in section 6 . 


\section{The Model}

Many left-right supersymmetric setups have been developed in the past. We adopt the configuration introduced in ref. [8]. The Standard Model left- and right-handed quarks (leptons) are embedded, together with their scalar superpartners, in $Q_{L}$ and $Q_{R}\left(L_{L}\right.$ and $\left.L_{R}\right)$ supermultiplets lying in the fundamental representation of the $\mathrm{SU}(2)_{L}$ and $\mathrm{SU}(2)_{R}$ gauge groups, respectively. The Higgs(ino) sector is constituted of two $\mathrm{SU}(2)_{L}$ triplets $\left(\Delta_{1 L}\right.$ and $\Delta_{2 L}$ with a $B-L$ charge $\left.Q_{B-L}=\mp 2\right)$, two SU $(2)_{R}$ triplets $\left(\Delta_{1 R}\right.$ and $\Delta_{2 R}$ with $Q_{B-L}=$ $\mp 2)$, two $\mathrm{SU}(2)_{L} \times \mathrm{SU}(2)_{R}$ bidoublets $\left(\Phi_{1}\right.$ and $\Phi_{2}$ not charged under the $B-L$ symmetry) and one gauge singlet $(S)$. As demonstrated in pioneering works, this configuration allows for a successful symmetry breaking of the $\mathrm{SU}(2)_{L} \times \mathrm{SU}(2)_{R} \times \mathrm{U}(1)_{B-L}$ gauge symmetry down to the $\mathrm{U}(1)$ electromagnetic gauge group, it includes a see-saw mechanism yielding the generation of the neutrino masses and it provides acceptable fermion masses and mixings with respect to data $[12-15]$.

Assuming a discrete $\mathbb{Z}_{3}$ symmetry, the superpotential only contains terms that are trilinear in the fields,

$$
\begin{aligned}
W= & Q_{L} y_{1}^{Q} \Phi_{1} Q_{R}+Q_{L} y_{2}^{Q} \Phi_{2} Q_{R}+L_{L} y_{1}^{L} \Phi_{1} L_{R}+L_{L} y_{2}^{L} \Phi_{2} L_{R}+L_{L} y_{3}^{L} \Delta_{2 L} L_{L} \\
& +L_{R} y_{4}^{L} \Delta_{1 R} L_{R}+\lambda_{L} S \Delta_{1 L} \cdot \Delta_{2 L}+\lambda_{R} S \Delta_{1 R} \cdot \Delta_{2 R}+\lambda_{1} S \Phi_{1} \cdot \Phi_{1}+\lambda_{2} S \Phi_{2} \cdot \Phi_{2} \\
& +\lambda_{12} S \Phi_{1} \cdot \Phi_{2}+\frac{1}{3} \lambda_{S} S^{3}
\end{aligned}
$$

where we refer to ref. [22] for details on the underlying (understood) index structure. In those notations, the various coupling strengths have been embedded into $3 \times 3$ Yukawa matrices $\left(y_{Q}^{i}\right.$ and $y_{L}^{j}$ with $i=1,2$ and $\left.j=1,2,3,4\right)$ and $\lambda$ parameters dictating the size of the Higgs self-interactions. The supersymmetry-breaking Lagrangian contains sfermion and gaugino mass terms as well as trilinear $(T)$ interactions whose structure is given by their superpotential counterparts.

The spontaneous symmetry-breaking mechanism proceeds in two steps. The $\mathrm{SU}(2)_{R} \times$ $\mathrm{U}(1)_{B-L}$ group is first broken to the hypercharge group and the two $\mathrm{SU}(2)_{R}$ Higgs triplets develop non-vanishing vacuum expectation values (vevs). In a second step, the electroweak symmetry is broken to electromagnetism and the bidoublet Higgs fields get non-zero vacuum expectation values. The vacuum state is characterized by the vevs of all the neutral components of the Higgs fields,

$$
\begin{aligned}
& \left\langle\Delta_{1 L}\right\rangle=\left\langle\left(\begin{array}{cc}
\frac{\Delta_{1 L}^{-}}{\sqrt{2}} & \Delta_{1 L}^{0} \\
\Delta_{1 L}^{-} & -\frac{\Delta_{1 L}^{-}}{\sqrt{2}}
\end{array}\right)\right\rangle=\left(\begin{array}{cc}
0 & \frac{v_{1 L}}{\sqrt{2}} \\
0 & 0
\end{array}\right), \quad\left\langle\Delta_{2 L}\right\rangle=\left\langle\left(\begin{array}{cc}
\frac{\Delta_{2 L}^{+}}{\sqrt{2}} & \Delta_{2 L}^{++} \\
\Delta_{2 L}^{0} & -\frac{\Delta_{2 L}^{+}}{\sqrt{2}}
\end{array}\right)\right\rangle=\left(\begin{array}{cc}
0 & 0 \\
\frac{v_{2 L}}{\sqrt{2}} & 0
\end{array}\right), \\
& \left\langle\Delta_{1 R}\right\rangle=\left\langle\left(\begin{array}{cc}
\frac{\Delta_{1 R}^{-}}{\sqrt{2}} & \Delta_{1 R}^{0} \\
\Delta_{1 R}^{-} & -\frac{\Delta_{1 R}^{-}}{\sqrt{2}}
\end{array}\right)\right\rangle=\left(\begin{array}{cc}
0 & \frac{v_{1 R}}{\sqrt{2}} \\
0 & 0
\end{array}\right), \quad\left\langle\Delta_{2 R}\right\rangle=\left\langle\left(\begin{array}{cc}
\frac{\Delta_{2 R}^{+}}{\sqrt{2}} & \Delta_{2 R}^{++} \\
\Delta_{2 R}^{0} & -\frac{\Delta_{2 R}^{+}}{\sqrt{2}}
\end{array}\right)\right\rangle=\left(\begin{array}{cc}
0 & 0 \\
\frac{v_{2 R}}{\sqrt{2}} & 0
\end{array}\right) \text {, } \\
& \left\langle\Phi_{1}\right\rangle=\left\langle\left(\begin{array}{cc}
\Phi_{1}^{0} & \Phi_{1}^{+} \\
\Phi_{1}^{-} & \Phi_{1}^{\prime 0}
\end{array}\right)\right\rangle=\left(\begin{array}{cc}
\frac{v_{d}}{\sqrt{2}} & 0 \\
0 & 0
\end{array}\right), \quad\left\langle\Phi_{2}\right\rangle=\left\langle\left(\begin{array}{cc}
\Phi_{2}^{\prime 0} & \Phi_{2}^{+} \\
\Phi_{2}^{-} & \Phi_{2}^{0}
\end{array}\right)\right\rangle=\left(\begin{array}{cc}
0 & 0 \\
0 & \frac{v_{u}}{\sqrt{2}}
\end{array}\right), \\
& \langle S\rangle=\frac{v_{S}}{\sqrt{2}},
\end{aligned}
$$


where all vevs are taken real and positive (as allowed by suitable field redefinitions). Although the $\Phi^{\prime 0}$ fields could also develop non-zero vacuum expectation values giving rise to $W / W_{R}$ mixing, these are strongly constrained by kaon data so that they are ignored. Moreover, the phase of $v_{S}$ cannot in principle be rotated away but we omit it for simplicity. The remaining vevs can be further constrained. Both the $v_{i R}$ vevs are imposed to be large by the masses of the $W_{R}$ and $Z_{R}$ gauge bosons. Furthermore, the smallness of the Standard Model neutrino masses and electroweak precision data require that the vevs developed by the $\mathrm{SU}(2)_{L}$ Higgs triplets $v_{i L}$ are negligible. ${ }^{1}$ Finally, the size of $v_{S}$ is related to the effective $\mu$-terms of the superpotential, and we consequently impose the hierarchy $v_{S}, v_{1 R}, v_{2 R} \gg v_{u, d} \gg v_{1 L} \approx v_{2 L} \approx 0$. For further references, we define $v_{1 R}^{2}+v_{2 R}^{2}=v_{R}^{2}, \tan \beta_{R}=v_{2 R} / v_{1 R}, v_{d}^{2}+v_{u}^{2}=v^{2}$ and $\tan \beta=v_{u} / v_{d}$ and we additionally assume $\lambda_{1}=\lambda_{2}=0$, analogously to refs. [22, 23].

The vacuum configuration of eq. (2.2) does not necessarily yield a minimum of the scalar potential [12]. In fact, the true global minimum is in general charge-breaking and the $\Delta_{1 R}^{--}$and $\Delta_{2 R}^{++}$fields develop a vev

$$
\left\langle\Delta_{i R}\right\rangle_{\mathrm{CB}}=\left(\begin{array}{cc}
0 & \frac{v_{i R}}{2} \\
\frac{v_{i R}}{2} & 0
\end{array}\right) \text { for } i=1,2,
$$

so that the $D$-term contributions to the scalar potential are minimized. We will further address this issue in section 3 .

In order to design viable left-right supersymmetric benchmark scenarios, we have implemented the model described above in the SARAH package [24-28] to automatically generate a numerical code based on the SPheno programme $[29,30] .^{2}$ Physical spectra are then calculated in several steps. One begins with the derivation of the soft-supersymmetry breaking masses from the tadpole equations at the tree level, which allows for the calculation of a tree-level mass spectrum. Next, one-loop corrections are evaluated in the $\overline{\mathrm{DR}}$ scheme and a one-loop-accurate mass spectrum is computed. As explained in the next section, we have modified the automated SARAH-SPheno procedure due to special features associated with the mass of the doubly-charged Higgs boson. Several analytical cross checks with the model implementation in FeynRules [31, 32] have been performed. For the evaluation of the LHC bounds performed in section 4, the SSP package [33, 34] has been used to scan randomly the parameter space of the model. Moreover, we have estimated $W_{R}$ signal cross sections by means of the MadGraph5_aMC@NLO programme [35] after having linked the UFO library [36] obtained from the SARAH implementation.

\section{The doubly-charged Higgs mass}

In the class of supersymmetric left-right symmetric models under consideration, we have four doubly-charged Higgs bosons, two of them being related to the $\mathrm{SU}(2)_{L}$ sector and two of them being related to the $\mathrm{SU}(2)_{R}$ one. While at the tree level, these fields do not mix, a

\footnotetext{
${ }^{1}$ Another acceptable choice implies that the $y_{3}^{L}$ couplings are tiny and the $y_{4}^{L}$ ones are large. This is however very unnatural with the left-right symmetry.

${ }^{2}$ The code can be obtained from the authors upon request.
} 

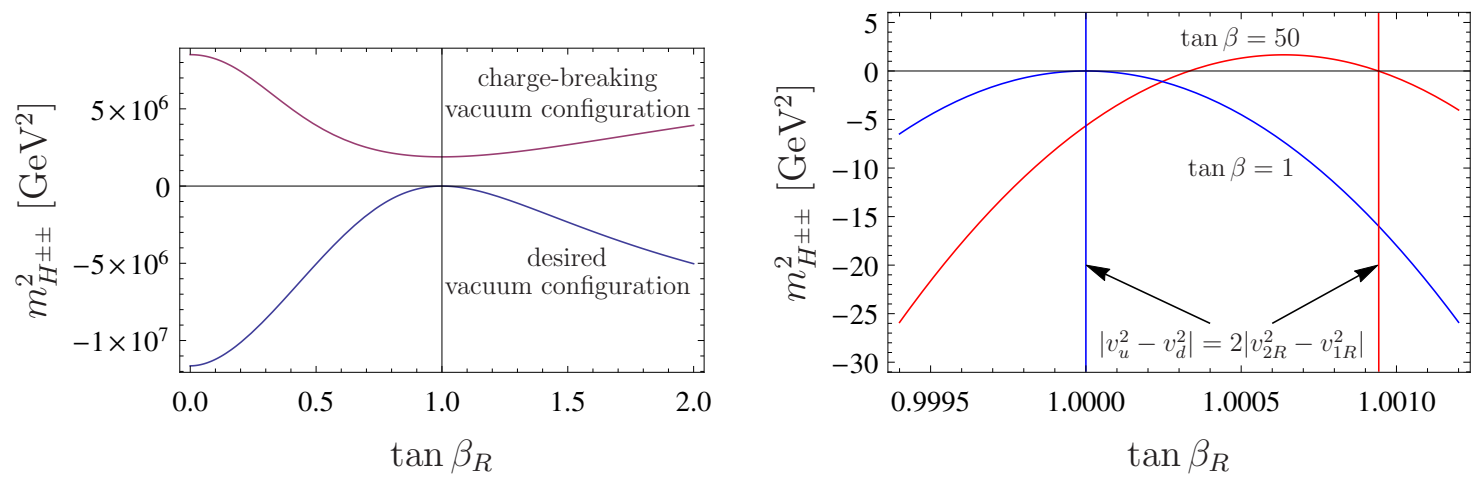

Figure 1. The squared mass eigenvalue of the lightest doubly-charged Higgs state $m_{H^{ \pm \pm}}^{2}$ for the desired vacuum configuration of eq. (2.2) (left panel, blue) and for the charge-breaking one of eq. (2.3) (left panel, purple) as a function of $\tan \beta_{R}$. We also zoom into the region with $\tan \beta_{R} \approx 1$ (right panel) and present results for the $\tan \beta=1$ (blue) and $\tan \beta=50$ (red) cases. Moreover, the condition $\left|v_{u}^{2}-v_{d}^{2}\right|>2\left|v_{2 R}^{2}-v_{1 R}^{2}\right|$ that is discussed in the text is illustrated by vertical lines. The relevant model parameters have been set to $v_{R}=5.5 \mathrm{TeV}, v_{S}=10 \mathrm{TeV}, \lambda_{R}=0.5, \lambda_{S}=-0.5$, $\lambda_{12}=-0.02$ and $T_{\lambda_{R}}=0$.

small mixing is induced at the loop level. It however does not lead to any observable effect so that the following discussion is solely focusing on the $\mathrm{SU}(2)_{R}$ sector. The mixing effects are nevertheless included in our numerical results.

\subsection{Tree-level results}

The squared mass matrix of the doubly-charged $\mathrm{SU}(2)_{R}$ Higgs bosons reads, at the tree level and in the $\left(\Delta_{1 R}^{--}, \Delta_{2 R}^{++*}\right)$ basis,

$$
m^{2}=\left(\begin{array}{cc}
D_{++}-\tan \beta_{R} F_{++} & F_{++} \\
F_{++} & -D_{++}-\cot \beta_{R} F_{++}
\end{array}\right) .
$$

In our conventions, the $D$-term and $F$-term contributions are respectively given by

$$
\begin{aligned}
& D_{++}=\frac{g_{R}^{2}}{2}\left[v_{d}^{2}-v_{u}^{2}+2\left(v_{2 R}^{2}-v_{1 R}^{2}\right)\right], \\
& F_{++}=\frac{\lambda_{R}^{2}}{2} v_{1 R} v_{2 R}+\frac{\lambda_{R} \lambda_{S}}{2} v_{S}^{2}-\frac{\lambda_{R} \lambda_{12}}{2} v_{d} v_{u}+\frac{T_{\lambda_{R}}}{\sqrt{2}} v_{S},
\end{aligned}
$$

where $g_{R}$ stands for the $\mathrm{SU}(2)_{R}$ gauge coupling. First, the form of this squared mass matrix implies that one of the physical states is massless in the gaugeless limit, i.e., when all gauge couplings are neglected. Next, once the contributions proportional to the gauge couplings are taken into account, the condition

$$
\left|v_{u}^{2}-v_{d}^{2}\right|>2\left|v_{2 R}^{2}-v_{1 R}^{2}\right|
$$

must be satisfied in order to render both doubly-charged Higgs squared mass eigenvalues positive [14]. Equivalently, this condition can be casted as $\tan \beta_{R}-1 \ll 1$ since we have imposed, for phenomenological reasons, that $v_{R} \gg v_{u}, v_{d}$ (see section 2). ${ }^{3}$

\footnotetext{
${ }^{3}$ The special case where $\tan \beta_{R}=1$ corresponds to a saddle point and not to a minimum of the potential.
} 


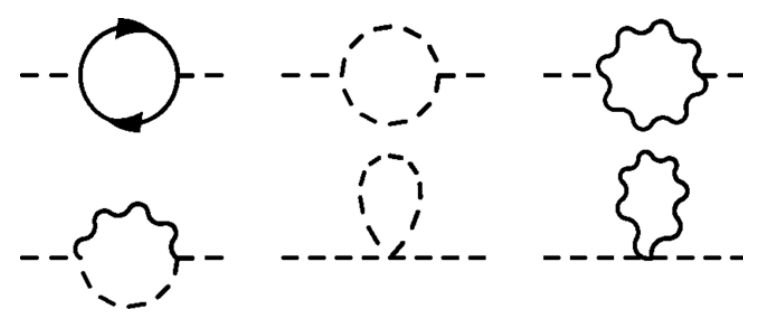

Figure 2. Generic one-loop diagrams contributing to the doubly-charged Higgs self-energy.

The condition of eq. (3.3) turns out to be extremely fine-tuned, as illustrated in figure 1 where we depict the dependence of the smallest mass eigenvalue $m_{H^{ \pm \pm}}^{2}$ on $\tan \beta_{R}$. In the left panel of the figure, we present numerical predictions for the desired vacuum configuration of eq. (2.2) (blue) as well as for the charge-breaking one of eq. (2.3) (purple). In contrast to the latter case where the squared mass is generally positive, there exists only a small region of the parameter space, featuring $\tan \beta_{R} \approx 1$, where the vacuum structure does not break electric charge invariance. In the right panel of figure 1 , we zoom into this region and study scenarios for which $\tan \beta_{R} \approx 1$. In the case where $\tan \beta=1$ (blue), or in other words when $\left|v_{u}^{2}-v_{d}^{2}\right|=0$, the condition of eq. (3.3) imposes that the lightest doubly-charged Higgs boson is either tachyonic or massless. In contrast, when $\tan \beta$ increases, a small window where the squared mass of the lightest doubly-charged Higgs boson is positive opens (red, for the example of $\tan \beta=50$ ). However, this configuration implies that one of the pseudoscalar Higgs bosons gets tachyonic [37].

As a consequence, it is impossible to construct, at the tree level, left-right supersymmetric setups that are phenomenologically viable. The only alternative option would be to consider solutions of the potential minimization equations featuring $R$-parity breaking vevs for the right-handed sneutrinos $[12,37]$.

\subsection{Results at the one-loop level}

The problems mentioned above are a tree-level artefact and can be solved by considering one-loop contributions. Attempts including quantum corrections derived either from the Coleman-Weinberg potential $[8,38]$ or within a Feynman diagrammatic approach [20] have shown that a charge-conserving minimum of the scalar potential could be obtained. A complete one-loop calculation however exhibits logarithmic terms with a negative argument resulting from contributions depending on the self-interactions of the doubly-charged Higgs bosons, and the subsequent imaginary part of the one-loop corrected potential is a sign of a dangerous unstable vacuum (see section 5). More precisely, these earlier works have argued that contributions stemming from the Majorana neutrino Yukawa coupling $y_{4}^{L}$ were sufficient to render the doubly-charged Higgs squared mass positive [20] and to make the vacuum configuration of eq. (2.2) a deeper minimum than the one of eq. (2.3) [8]. However, in this last case, the right slepton soft squared masses $m_{L_{R}}^{2}$ must be negative, which further implies large one-loop gaugino contributions to the slepton mass eigenvalues to avoid charge and $R$-parity breaking. 

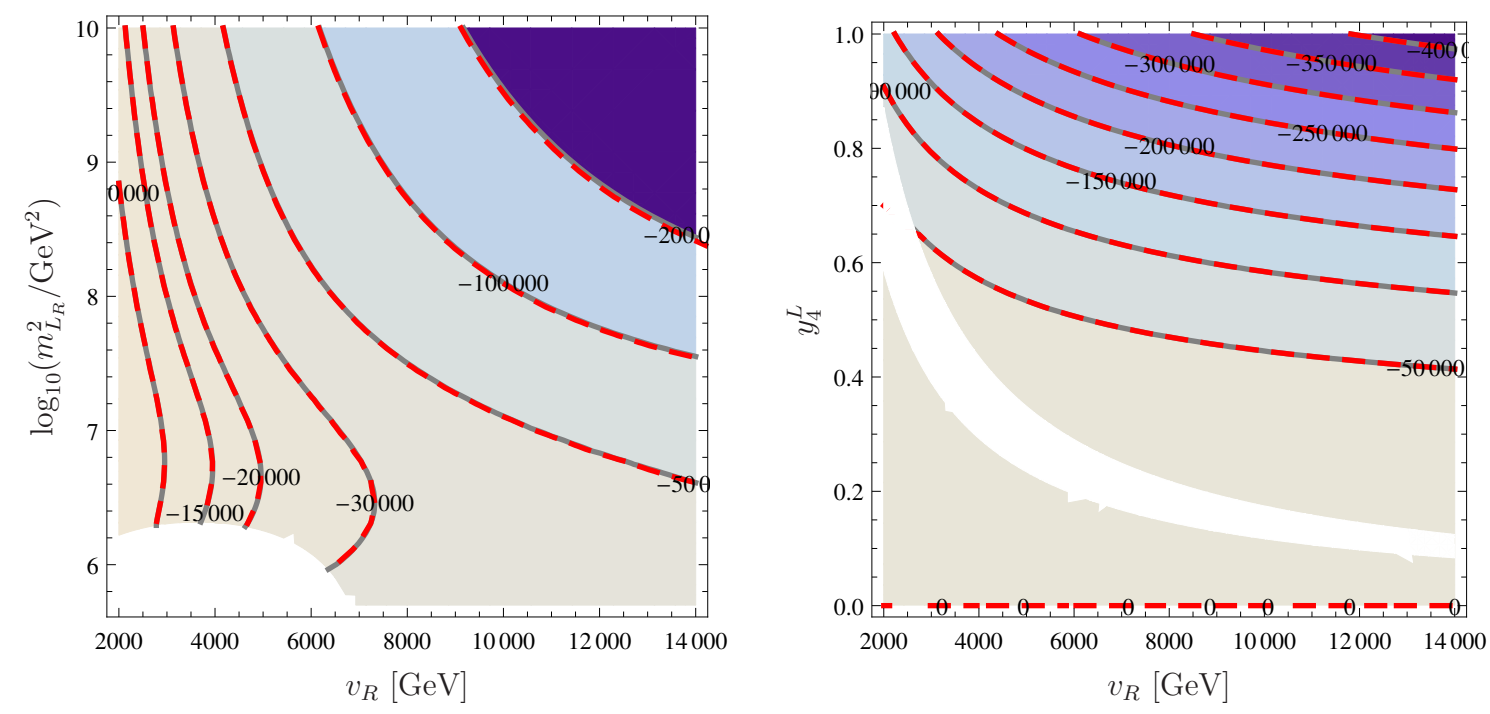

Figure 3. Comparison of our calculation of the one-loop corrections from $y_{4}^{L}$, evaluated in the gaugeless limit and for one generation of right-handed neutrinos (red dashed), to the results of ref. [20] (grey). The contour lines represent $m_{H^{ \pm \pm}}^{2}$ isomass lines in $\mathrm{GeV}^{2}$. We present results in the $\left(v_{R}, \log _{10}\left(m_{L_{R}}^{2} / \mathrm{GeV}^{2}\right)\right)$ plane with $y_{4}^{L}=0.4$ (left panel) and in the $\left(v_{R}, y_{4}^{L}\right)$ plane with $m_{L_{R}}^{2}=2 \cdot 10^{6} \mathrm{GeV}^{2}$ (right panel). In both cases, the remaining relevant parameters are fixed to $\lambda_{R}=0.4, \tan \beta_{R}=1.02$ and $v_{S}=10 \mathrm{TeV}$ and the white areas are regions where one of the right sneutrinos is tachyonic.

In this work, we have extended the Feynman-diagram-based calculations of ref. [20], the latter having been achieved in the gaugeless limit, for one generation of right-handed neutrinos and considered only diagrams depending on $y_{4}^{L}$. We have instead computed all one-loop contributions to the doubly-charged Higgs boson mass, the associated Feynman diagrams being presented in figure 2 . In figure 3 , we start by numerically comparing our results, evaluated in the appropriate limit, to those of ref. [20] and demonstrate that a good agreement has been found. ${ }^{4}$ In the parameter space regions probed on the figure, the soft slepton mass $m_{L_{R}}^{2}$ and the effective supersymmetric Higgs mass parameter $\mu_{R}^{\mathrm{eff}}=\lambda_{R} v_{S} / \sqrt{2}$ are not too large, which leads to a doubly-charged Higgs boson that is still tachyonic. The loop corrections are even in this case negative and thus counterproductive to restore a vacuum state that conserves the electric charge. Only for large values of $\lambda_{R} v_{S}$ (not shown on the figure), a positive contribution could emerge. This requirement however also yields a significant $C P$-splitting of the right sneutrinos, so that large values for $m_{L_{R}}^{2}$ are as well required to prevent them from being tachyonic and breaking $R$-parity. Assuming that all sfermions have similar masses, realistic setups of the class of models under study are thus unlikely to be observable from standard sfermion searches at the LHC.

As shown in the rest of this section, the situation improves when the complete one-loop result is considered. Technically, our predictions rely on the SARAH-SPheno procedure for generating the particle spectrum. In the usual running mode of both programmes, the

\footnotetext{
${ }^{4}$ The authors of ref. [20] have confirmed a typo in the first line of their eq. (138) in which the ' +1 ' should be read outside of the logarithm.
} 

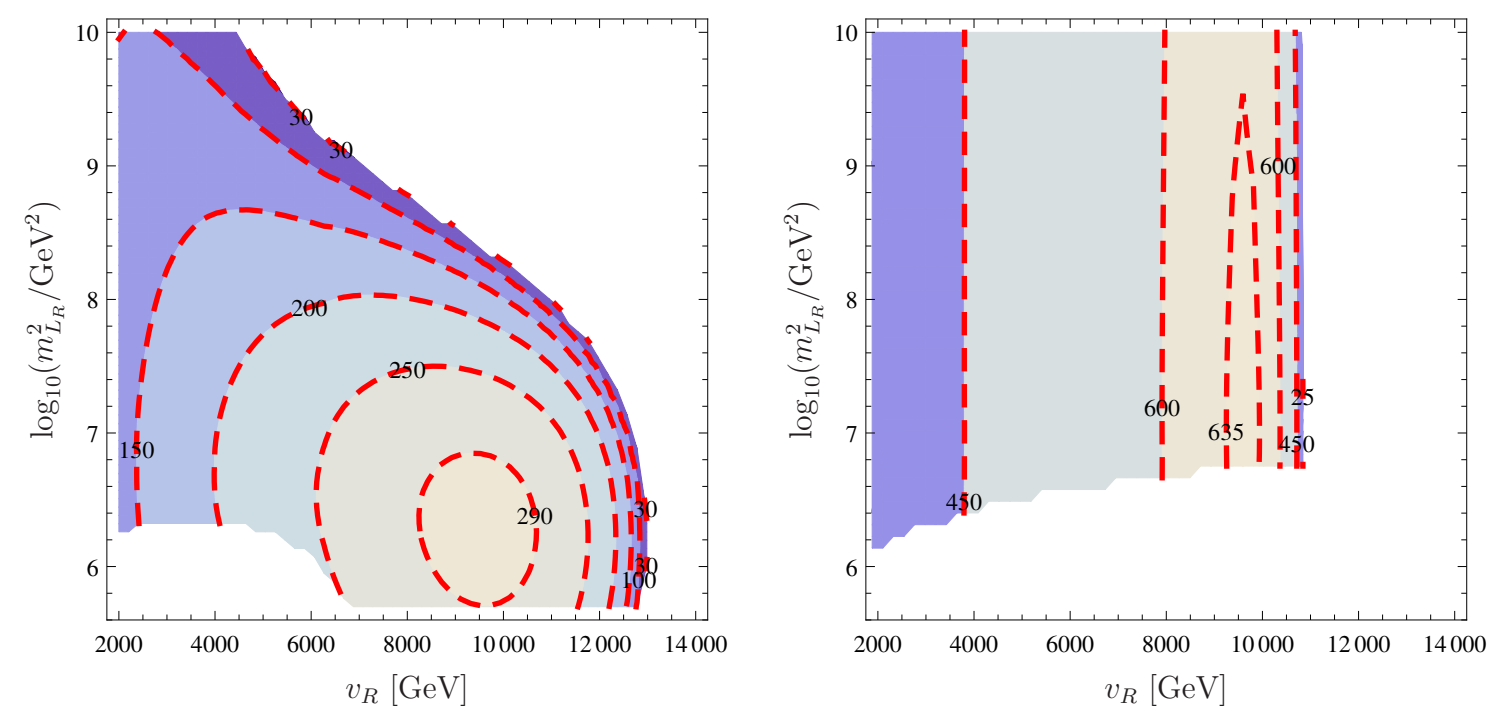

Figure 4. Contours of $m_{H^{ \pm \pm}}$[in $\mathrm{GeV}$ ] including the complete one-loop calculation in the $\left(v_{R}, \log _{10}\left(m_{L_{R}}^{2} / \mathrm{GeV}^{2}\right)\right)$ plane for a scenario featuring the same setup as the left panel of figure 3 (left), as well as for a scenario featuring a smaller $y_{4}^{L}=0.1$ and a larger $\lambda_{R}=0.9$ value (right).

tree-level $\overline{\mathrm{DR}}$-masses are used for the particles running into the loop diagrams. In the leftright supersymmetric models under consideration, this leads to problematic $\log \left(m_{H^{ \pm \pm}}^{2} / Q^{2}\right)$ terms as the lightest doubly-charged Higgs boson is tachyonic at the tree level. To avoid this issue, we have modified the SARAH-SPheno procedure in a way that is inspired by the on-shell scheme, and inserted one-loop corrected masses into the loops. Iteratively, this consists of:

- first iteration: $m_{[1]}^{2}=m^{2}\left(m_{[0]}^{2}\right)$ using $m_{[0]}^{2}=\left|\left[m_{H^{ \pm \pm}}^{2}\right]_{\text {tree-level }}\right|$;

- $m_{[k]}^{2}=m^{2}\left(m_{[k-1]}^{2}\right)$ until $\frac{\left|m_{[k]}-m_{[k-1]}\right|}{m_{[k]}}<\epsilon \ll 1$.

We have fixed $\epsilon=10^{-4}$ so that four iterations are generally necessary for any benchmark scenario. Moreover, the initial value $m_{[0]}^{2}$ is in principle arbitrary so that we could have chosen $m_{[0]}^{2}=0$. We have verified the invariance of the predictions with respect to this choice. Although our prescription breaks gauge invariance at the two-loop level, the associated effects are expected to be significantly smaller than the genuine two-loop contributions and thus under good control. Here and in the following sections $m_{H^{ \pm \pm}}$denotes the mass of the lightest doubly-charged Higgs boson.

In figure 4, we show predictions for the $H^{ \pm \pm}$mass obtained when using our complete one-loop calculation. The results are presented in $\left(v_{R}, \log _{10}\left(m_{L_{R}}^{2} / \mathrm{GeV}^{2}\right)\right)$ planes, first (left panel of the figure) when all the other model parameters are fixed as in the left panel of figure 3. Although both the tree-level and the approximate one-loop predictions yield tachyonic doubly-charged Higgs bosons, the fully-one-loop-corrected mass is positive and of the order of a few hundreds of GeV. Moreover, the dependence on $m_{L_{R}}^{2}$ is explained by the $y_{4}^{L}$-dependent contributions. In the right panel of figure 4 , we increase the value of $\lambda_{R}$ and 
decrease the one of $y_{4}^{L}$ so that the dependence on $m_{L_{R}}^{2}$ almost vanishes and the one-loopcorrected $H^{ \pm \pm}$mass reaches values beyond $500 \mathrm{GeV}$. The most important contributions to the full one-loop result consist of positive corrections arising from $W_{R} /$ singly-charged Higgs boson and doubly-chargino/neutralino loop-diagrams, as well as from negatively contributing $y_{4}^{L}$-induced loop-diagrams, singly-charginos and doubly-charged Higgs bosons.

The parametric dependence of the loop contributions is highly non-trivial because of the large number of different contributing sectors. However, some generic features can be extracted using the information given in figure 5, where we add successively different loop contributions for an exemplary benchmark scenario. We start by adding to the tree-level results the $y_{4}^{L}$-dependent contributions which have already been discussed: they become negative for large values of $y_{4}^{L}$ and $m_{L_{R}}^{2}$.

In order to describe the additional contributions that will be further added, we recall that the structure of the relevant chargino and neutralino couplings reads

$$
\begin{aligned}
\Gamma_{\tilde{\chi}_{R}^{++}}^{L} \tilde{\chi}_{j}^{0} H_{k}^{--} & =-i\left(\lambda_{R} U_{j, \tilde{S}}^{0, *} Z_{k, \Delta_{1 R}^{--}}^{--}+\sqrt{2}\left(g_{B L} U_{j, \tilde{B}}^{0, *} Z_{k, \Delta_{2 R}^{++, *}}^{--}+g_{R} U_{j, \tilde{W}_{R, 3}}^{0, *} Z_{k, \Delta_{2 R}^{++*}}^{--}\right)\right), \\
\Gamma_{\tilde{\chi}_{R}^{++} \tilde{\chi}_{j}^{0} H_{k}^{--}}^{R} & =-i\left(\lambda_{R} U_{j, \tilde{S}_{k, \Delta_{2 R}}^{0} Z_{k, *}^{--}}^{++,} \sqrt{2}\left(g_{B L} U_{j, \tilde{B}}^{0} Z_{k, \Delta_{1 R}^{--}}^{--}+g_{R} U_{j, \tilde{W}_{R, 3}}^{0} Z_{k, \Delta_{1 R}^{--}}^{--}\right)\right), \\
\Gamma_{\tilde{\chi}_{i}^{+} \tilde{\chi}_{j}^{+} H_{k}^{--}}^{L} & =i \sqrt{2} g_{R} Z_{k, \Delta_{2 R}^{+, *}}^{--}\left(U_{i, \tilde{W}_{R}^{+}}^{+, *} U_{j \tilde{\Delta}^{+}}^{+, *}+U_{i \tilde{\Delta}^{+}}^{+, *} U_{j, \tilde{W}_{R}^{+}}^{+, *}\right), \\
\Gamma_{\tilde{\chi}_{i}^{+} \tilde{\chi}_{j}^{+} H_{k}^{--}}^{R} & =-i \sqrt{2} g_{R} Z_{k, \Delta_{1 R}^{--}}^{--}\left(U_{i, \tilde{W}_{R}^{+}}^{-} U_{j, \tilde{\Delta}^{+}}^{-}+U_{i \tilde{\Delta}^{+}}^{-} U_{j, \tilde{W}_{R}^{+}}^{-}\right),
\end{aligned}
$$

where we have parameterised the vertices as $\Gamma=\Gamma^{L} P_{L}+\Gamma^{R} P_{R}$. In our notations, $U^{0}, U^{ \pm}$ and $Z^{--}$are the mixing matrices of the neutralinos, charginos and doubly-charged Higgs bosons respectively, whose elements are written as $U_{i, X}$ and $Z_{i, X}$. These matrix elements hence denote the $X$ field component of the $i^{\text {th }}$ mass eigenstate. We also introduce a set of vector and Higgs boson interactions whose strengths are given by

$$
\begin{aligned}
& \Gamma_{H_{j}^{--} W_{R}^{+} W_{R}^{+}}=-i \sqrt{2} g_{R}^{2}\left(v_{1 R} Z_{j, \Delta_{1 R}^{--}}^{--}+v_{2 R} Z_{j, \Delta_{2 R}^{++}}^{--}\right), \\
& \Gamma_{H_{j}^{--} H_{k}^{+} W_{R}^{+}}=-i\left(g_{R} Z_{k, \Delta_{1 R}^{-, *}}^{-, Z_{j, \Delta_{1 R}^{--}}^{--}}+g_{R} Z_{k, \Delta_{2 R}^{+}}^{-} Z_{j, \Delta_{2 R}^{++, *}}^{--}\right), \\
& \Gamma_{H_{i}^{--} H_{j}^{+} H_{k}^{+}}=\frac{i}{\sqrt{2}} g_{R}^{2}\left(2\left(v_{2 R} Z_{j, \Delta_{2 R}^{+}}^{-} Z_{k, \Delta_{2 R}^{+}}^{-} Z_{i, \Delta_{2 R}^{++, *}}^{--}+v_{1 R} Z_{j, \Delta_{1 R}^{-, *}}^{-} Z_{k, \Delta_{1 R}^{-}, *}^{-} Z_{i, \Delta_{1 R}^{--}}^{--}\right)\right. \\
& \left.-\left(Z_{j, \Delta_{2 R}^{+}}^{-} Z_{k, \Delta_{1 R}^{-* *}}^{-}+Z_{j, \Delta_{1 R}^{-, *}}^{-} Z_{k, \Delta_{2 R}^{+}}^{-}\right)\left(v_{2 R} Z_{i, \Delta_{1 R}^{--}}^{--}+v_{1 R} Z_{i, \Delta_{2 R}^{++, *}}^{--}\right)\right),
\end{aligned}
$$

the momentum and metric dependence being omitted from the Feynman rules.

Beside the $y_{4}^{L}$ contributions, a set of self-energy diagrams also contribute negatively. These involve either two singly-charged Higgs bosons, the doubly-charged Higgs boson and a vector boson or two singly-charged charginos. The sign of the latter, where one would have naively expected a positive contribution, can be understood from the large mixing between the gaugino and the singly-charged Higgs bosons of the $\mathrm{SU}(2)_{R}$ sector. The contributions to the diagonal entries of the doubly-charged Higgs mass matrix are 


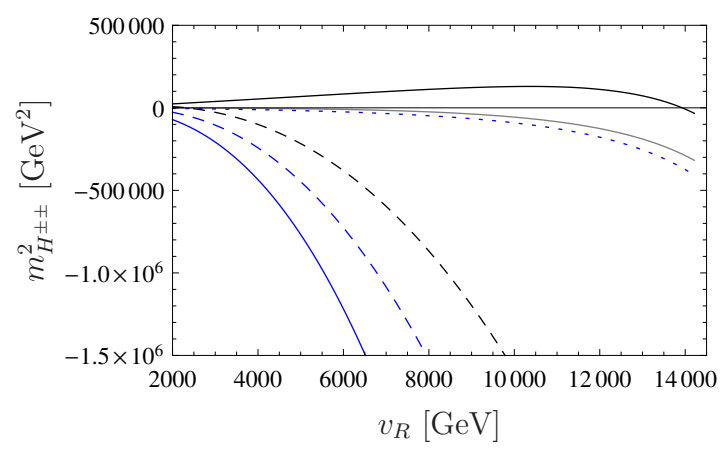

Figure 5. Dependence of $m_{H^{ \pm \pm}}^{2}$ on the $\mathrm{SU}(2)_{R^{-}}$-breaking scale $v_{R}$. The lines correspond to to the tree level prediction (grey solid) and add then successively different contributions: tree-level $+y_{4}^{L}$-dependent (s)lepton and (s)neutrino contributions (blue dotted) + singly/doubly charged Higgses and neutral gauge bosons (blue dashed), + chargino contributions (blue solid), + neutralinos and doubly-charged Higgsinos (black dashed). The full result (black solid) finally contains also $W_{R} / H^{ \pm}$contributions. The employed benchmark scenario is defined by $\lambda_{R}=0.4, \tan \beta_{R}=1.02$, $m_{L_{R}}^{2}=2 \cdot 10^{6} \mathrm{GeV}, y_{4}^{L}=0.25$ and features one generation of right-handed neutrinos.

proportional to $\left(\left|\Gamma_{\tilde{\chi}_{i}^{+} \tilde{\chi}_{j}^{+} H_{k}^{--}}^{L}\right|^{2}+\left|\Gamma_{\tilde{\chi}_{i}^{+} \tilde{\chi}_{j}^{+} H_{k}^{--}}^{R}\right|^{2}\right) m_{H^{ \pm \pm}}^{2}$ whereas the contributions to its offdiagonal entries are proportional to $\left(\Gamma_{\tilde{\chi}_{i}^{+} \tilde{\chi}_{j}^{+} H_{k}^{--}}^{L}\right)\left(\Gamma_{\tilde{\chi}_{i}^{+} \tilde{\chi}_{j}^{+} H_{l}^{--}}^{R}\right)^{*} m_{\tilde{\chi}_{i}^{+}} m_{\tilde{\chi}_{j}^{+}}$. The masses of the relevant charginos are however proportional to $v_{R}$ and the wino soft supersymmetrybreaking mass so that they are in general much larger than $m_{H^{ \pm \pm}}$. The contributions to the off-diagonal entries of the doubly-charged Higgs mass matrix turn therefore out to be much larger than those to the diagonal ones. After diagonalisation, this yields a negative contribution to $m_{H^{ \pm \pm}}$.

The main positive contribution to the doubly-charged Higgs mass are loop diagrams containing a neutralino and a doubly-charged higgsino. The difference with the singlychargino case depicted above stems from the singlino component of the neutralinos. In the respective entries of the mass matrix, the product $\left(\Gamma_{\tilde{\chi}_{R}^{+}}^{L / R} \tilde{\chi}_{j}^{0} H_{k}^{--}\right)\left(\Gamma_{\tilde{\chi}_{R}^{++} \tilde{\chi}_{j}^{0} H_{l}^{--}}^{L / R}\right)^{*}$ involves $\lambda_{R^{-}}$dependent terms whose sign is different from the one of the gauge contributions. These terms will hence dominate for large values of $\lambda_{R}$. Additionally, the $W_{R} / H^{+}$loop diagrams also contribute positively to the diagonal entries of the $H^{ \pm \pm}$mass matrix.

Eventually, the negative growth of the tree-level contribution to the $H^{ \pm \pm}$mass (grey line in figure 5) with increasing $v_{R}$ cannot be compensated anymore so that the $H^{ \pm \pm}$gets again tachyonic for large enough $v_{R}$. This scale turns to be $v_{R} \simeq 14 \mathrm{TeV}$ for the example of figure 5 .

Both LHC collaborations have set bounds on the mass of the doubly-charged Higgs boson. These limits however strongly depend on the final state in which the doublycharged Higgs boson decays into $[21,39]$. In this way, $m_{H^{ \pm \pm}}$is constrained to be larger than $204 \mathrm{GeV}, 459 \mathrm{GeV}, 396 \mathrm{GeV}$ and $444 \mathrm{GeV}$ for $\tau \tau, \mu \mu$, $e \mu$ or $e$ final states, respectively. As shown in figure 4 , there is usually a large range of $v_{R}$ values, while keeping all other parameters fixed, for which these LHC constraints are satisfied. However, $v_{R}$ also sets the mass scale of the $W_{R}$ boson so that one can combine these constraints with LHC limits on the $W_{R}$ mass (see section 4 ). 


\section{LHC bounds}

The model parameters entering in the above computations can also be experimentally constrained by searching for the rest of the particle spectrum. We focus here on the electroweak part, i.e., extra gauge bosons and right-handed neutrinos, updating the results first given in the report of the 2013 Les Houches workshop [23].

In the left-right supersymmetric setup under study in this work, the extra $Z_{R}$ boson is always heavier than its $W_{R}$ charged counterpart. We hence focus on the latter since it is more constraining. In general, a $W_{R}$ boson can decay into pairs of fermions as the Standard Model $W$ boson, into pairs of Standard Model bosons, into pairs of sfermions, into a chargino and a neutralino final state, and into a charged lepton and a right-handed neutrino. Therefore, one can experimentally look for it in several search channels, each setting its own bounds. The seemingly most stringent one is typically set by investigating the signature of a charged lepton and missing energy, assuming that the new $W_{R}$ boson decays into a lepton and a low-mass neutrino which escapes detection. However, in a left-right scenario this bound does not apply. When neutrino data is explained by a seesaw mechanism, the decay of the $W_{R}$ into a lepton and a low-mass neutrino is generally suppressed by the small neutrino mixing, while the $W_{R} \rightarrow \ell \nu_{R}$ mode will typically give rise to more complicated final states. Before focusing on the latter channel, more involved due to the unknown right-handed neutrino mass, we review the simpler searches with hadronic two-body final states, i.e., $W_{R} \rightarrow j j$ and $W_{R} \rightarrow t b$. In the first case, we can reinterpret the CMS inclusive dijet search of ref. [40]. In the second case, a direct comparison to the CMS search for $W_{R}$ bosons in the $t b$ channel of ref. [41] is in order. We do not consider the ATLAS counterparts because they are less sensitive. The above searches do apply to left-right models since the coupling strength of the $W_{R}$ boson to a pair of quarks is equal to that of the Standard Model $W$ boson, given that $g_{R}=g_{L}$ holds. Their reinterpretation is done by comparing the $p p \rightarrow W_{R} \rightarrow j j(t b)$ cross sections evaluated with the MadGraph5_aMC@NLO programme [35] to the excluded cross sections as a function of the $W_{R}$ mass. More precisely, our predictions have been evaluated by convoluting leading-order squared matrix elements with the CTEQ6L1 set of parton densities [42] and include a $K$-factor of 1.23 and 1.2 for $p p \rightarrow j j$ and $p p \rightarrow t b$, respectively.

In figure 6 the cross sections excluded by CMS are compared to those computed in our model. In the right panel of the figure, we observe that considering the full model spectrum decreases the constraint by typically $50-100 \mathrm{GeV}$, due to the appearance of extra decay modes for the $W_{R}$ that are not considered in the simplified models adopted in the experimental analyses. These extra channels get the $W_{R}$ decay width proportionally larger, slightly suppressing the branching ratios into the final states that are searched for. The spread in the model prediction reflects the scan over the parameter space. The dijet final state, shown in the left panel of the figure, is the one setting the tightest bound, $M_{W_{R}} \gtrsim 2.3 \mathrm{TeV}$. The corresponding bound on the right-handed vev is given by $v_{R} \gtrsim 4.9 \mathrm{TeV}$. A value of the $W_{R}$ boson mass between 1.8 and $2.0 \mathrm{TeV}$, which could be allowed by the dijet search depending on the specific choice of the input parameters, is fully excluded when the $t b$ search is also considered. 

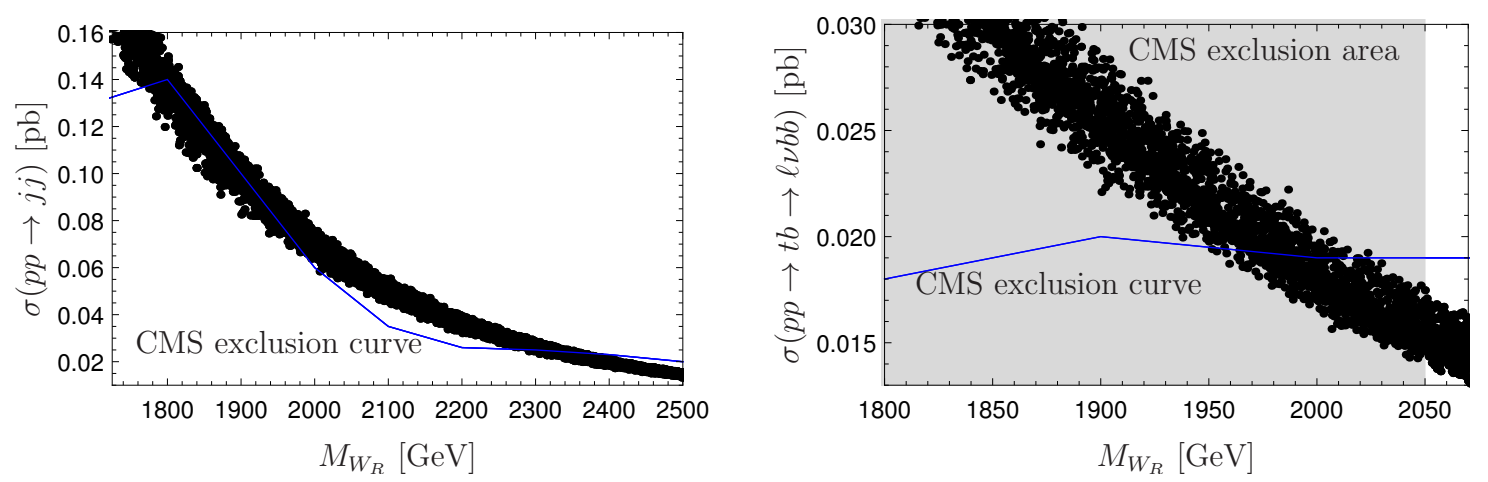

Figure 6. Bounds on the $W_{R}$ boson obtained from the CMS dijet search (left) and analysis of $t b$ leptonic events $(\ell=e, \mu, \tau)$ (right). The CMS exclusion curves are taken from ref. [40] and ref. [41], respectively. In the latter search, CMS puts a direct bound of $M_{W_{R}}>2.05 \mathrm{TeV}$, while no such bound exists in the former CMS analysis.

We move now to the constraints in the $W_{R} \rightarrow \ell \nu_{R}$ process. It is usually assumed in left-right symmetric models that the right-handed neutrinos can only decay via the $W_{R}$ boson, which can be either on- or off-shell, with the subsequent decay of the latter into two jets in two thirds of the cases. Therefore, footprints of the left-right models are searched for in the $p p \rightarrow \ell \ell j j(\ell=e, \mu)$ final state. This is used by experimental collaborations to set strict bounds on a combination of masses of $\nu_{R}$ and $W_{R}$, as, e.g., in ref. [43]. As shown in refs. [23, 44], this simplified assumption can sometimes be too restrictive. This is particularly true in the left-right supersymmetric models under consideration, where new two-body decay channels for the $\nu_{R}$ can be present, such as $\nu_{R} \rightarrow \ell^{\mp} H^{ \pm}$and decays into a neutral (charged) slepton and a chargino (neutralino). Furthermore, several other decay modes for the $W_{R}$ boson exist, thereby providing new three-body decays for the right-handed neutrinos. This is pictorially shown in figure 7 .

All these new decay modes for the right-handed neutrinos have the net effect of reducing its branching ratio into the search channel of interest, i.e., $\nu_{R} \rightarrow \ell j j$. Two main effects are visible. The right-handed neutrino prefers to decay into the lightest charged Higgs, if this channel is kinematically viable, with the subsequent $H^{+} \rightarrow t \bar{b}$ decay. However, this decay is possible only due to the (small) right-handed Higgs triplet component of the lightest charged Higgs. Therefore it is dominant only when the $W_{R}$ needs to be far off-shell for the three-body decays to occur, otherwise the latter will dominate, as happens for large masses of the right-handed neutrino. We observe the second effect on the figure. The presence of extra decay modes for the $W_{R}$ (mainly charginos and neutralinos) further suppresses the searched channel, which in the experimental approximation has a constant branching ratio of $67 \%$. However, the branching ratio $\operatorname{BR}\left(\nu_{R} \rightarrow \ell^{\mp} H^{ \pm}\right)$depends on the right-handed neutrino mass as well as on the singly-charged Higgs mass. It can get up to $\mathcal{O}(80 \%)$ for very light singly-charged Higgs bosons and right-handed neutrinos and conversely heavy $W_{R}$ bosons. 


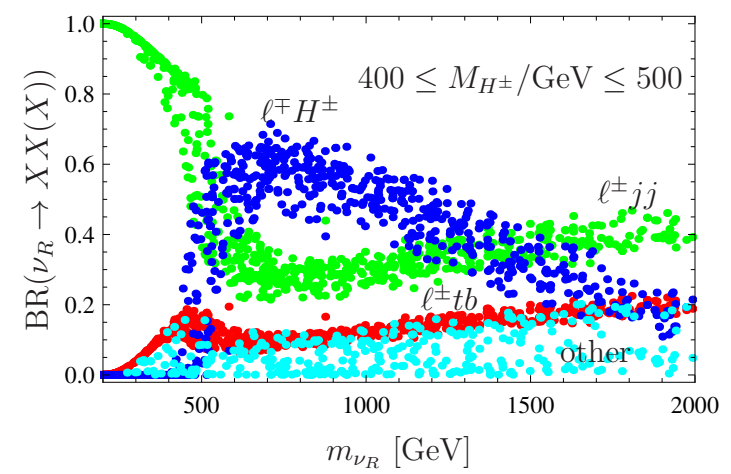

Figure 7. Right-handed neutrino branching ratios for a charged-Higgs mass of $400 \mathrm{GeV}<M_{H^{ \pm}}<$ $500 \mathrm{GeV}$. 'Other' refers to all other three-body decays via an off-shell $W_{R}$ boson.
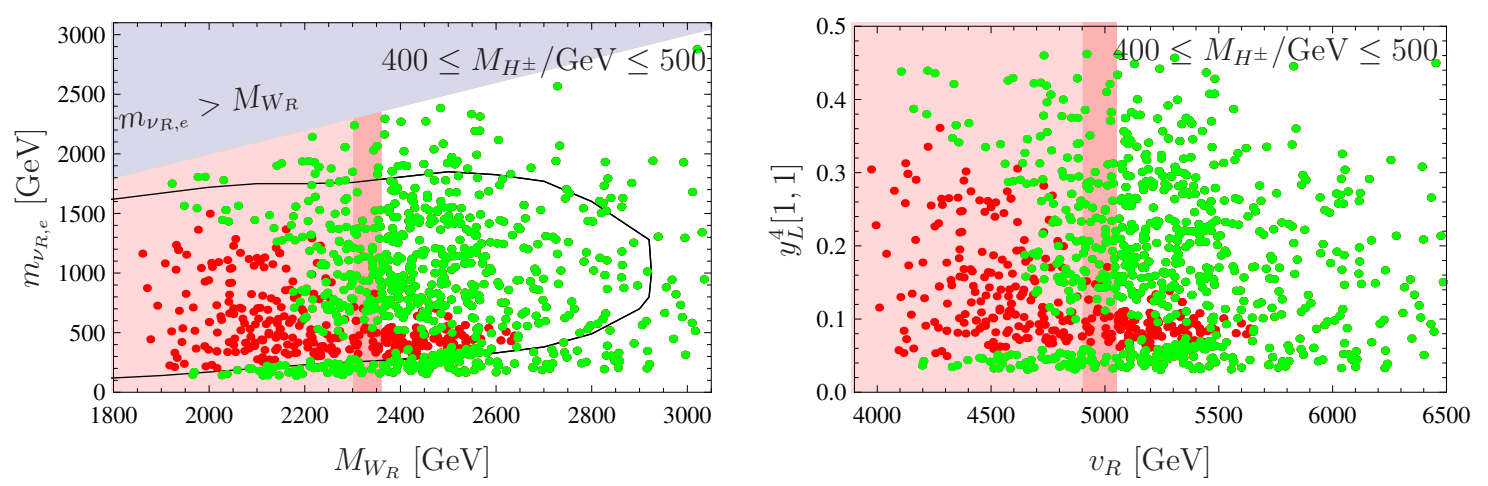

Figure 8. Allowed (green) and excluded (red) points from the CMS search for $W_{R}$ and $\nu_{R}$ in the eejj channel for a lightest right-handed neutrino of electron flavour (of mass $m_{\nu_{R, e}}$ ), for $400<$ $M_{H^{ \pm}} / \mathrm{GeV}<500$, in the $\left(m_{\nu_{R e}}, M_{W_{R}}\right)$ plane (left) and $\left(y_{L}^{4}[1,1], v_{R}\right)$ plane (right). The CMS exclusion curve (in black in the left panel of the figure) is taken from ref. [43]. The red shaded area represents the dijet bound.

As done previously for the hadronic two-body decays of the $W_{R}$ boson, we now reinterpret the CMS bounds on the $W_{R} \rightarrow \ell \nu_{R} \rightarrow \ell \ell j j$ channel of ref. [43]. Figures 8-9 display the allowed points (green dots) as well as the excluded points (red dots) when the considered right-handed neutrino is of electron and of muon flavour, respectively, in the $\left(m_{\nu_{R}}, M_{W_{R}}\right)$ plane on the left panel of the figure, and in the $\left(y_{L}^{4}, v_{R}\right)$ plane on its right panel.

We have found that the bounds set by CMS are generally too strong to be naively imposed in our left-right supersymmetric setup, and quite large areas within the excluded regions survive, especially for an electron right-handed neutrino. Investigating also figure 7, we identify three regions where exist parameter configurations which are allowed. First, for very light right-handed neutrinos, its decay products are too few energetic and appear below threshold. This consists of the lower region, below the black curve, in figures 8-9. Next, the region at high masses for both the $W_{R}$ and the $\nu_{R}$, that contains allowed points because of extra two-body decays of the $W_{R}$ that suppress the search channels. Last, the bulk area in figure 8 is now allowed, contrary to the CMS results, because of the suppression of the associated cross section due to the $\nu_{R} \rightarrow \ell^{\mp} H^{ \pm}$decay channel which is dominant 

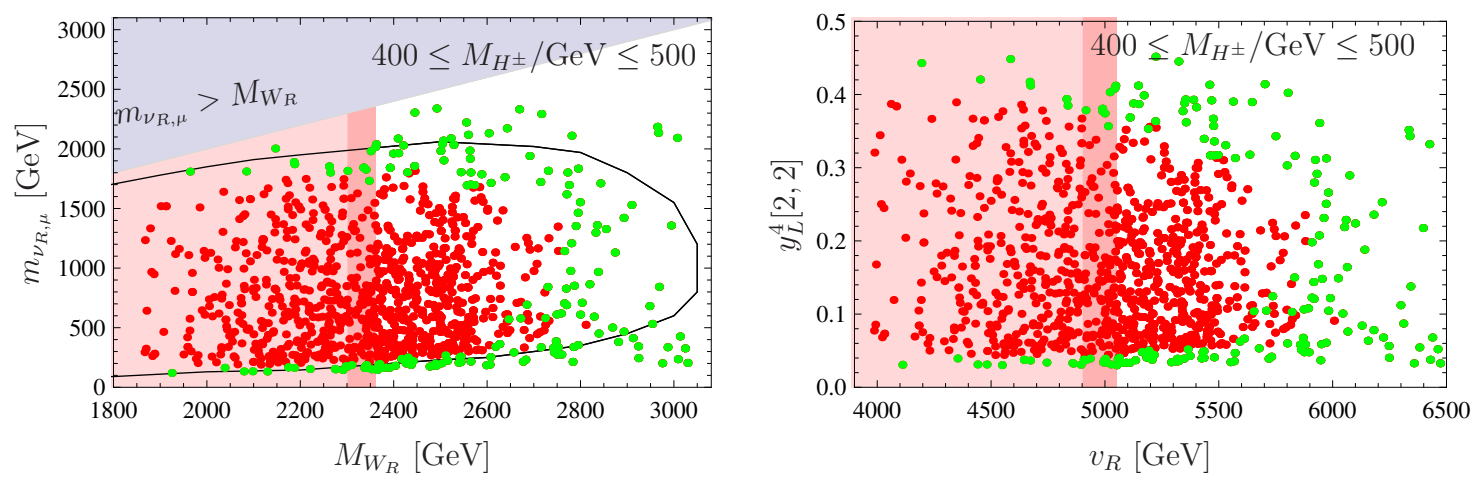

Figure 9. Same as in figure 8, for a lightest right-handed neutrino of a muon flavour (of mass $m_{\nu_{R, \mu}}$ ).

for moderate $\nu_{R}$ masses when such channel is kinematically open. Such an area does not appear for a muonic right-handed neutrino because of the much stronger CMS bounds. In fact, a fluctuation of about $3 \sigma$ has been found in the eejj CMS search channel, for $M_{W_{R}} \simeq 2.0 \mathrm{TeV}$, which our model can fit, as noted in refs. [45, 46] for similar cases.

\section{Analysis of the vacuum stability}

In section 3 we have shown that loop corrections have the power to switch the $\mathrm{SU}(2)_{L} \times$ $\mathrm{U}(1)_{Y}$ breaking saddle point into a minimum and to give a positive squared mass for $H^{ \pm \pm}$. Having now a local minimum with a proper breaking of $\mathrm{SU}(2)_{L} \times \mathrm{U}(1)_{Y}$ does not necessarily imply that this is also the global minimum of the theory. In particular, it is not obvious that it is deeper lying than the charge-breaking minimum of eq. (2.3) after taking into account consistently all loop effects. For further references, we denote in the following the vacuum configuration with the desired symmetry breaking pattern as 'DSB' and the charge-breaking vacuum as 'CB'.

The tadpole conditions cannot be solved analytically at the one-loop level so that one has to rely on numerical methods. However, the main features of the vacuum configuration can already be understood by considering a simplified model where we focus on the $\mathrm{SU}(2)_{R} \times \mathrm{U}(1)_{B-L}$ gauge sector, including only the two $\mathrm{SU}(2)_{R}$ Higgs triplets $\Delta_{1 R}$ and $\Delta_{2 R}$ as well as one generation of right-handed (s)leptons. The corresponding superpotential is given by

$$
W=y_{4}^{L} L_{R}^{T} \Delta_{1 R} \epsilon L_{R}+\lambda_{R} S \operatorname{Tr}\left(\Delta_{1 R} \Delta_{2 R}\right)+\frac{1}{3} \lambda_{S} S^{3},
$$

where we have written down the full $\mathrm{SU}(2)$ matrix structure for the first term using $L_{R}=\left(\nu_{R}^{c}, \ell_{R}^{c}\right)^{T}$ as well as the $\epsilon$ tensor defined by $\epsilon_{12}=-\epsilon_{21}=1$. The tree-level scalar potential $V_{0}$ reads

$$
V_{0}=V_{F}+V_{D}+V_{\text {soft }}
$$


with

$$
\begin{aligned}
V_{F}= & \lambda_{R}^{2}|S|^{2}\left(\operatorname{Tr}\left(\Delta_{1 R}^{\dagger} \Delta_{1 R}\right)+\operatorname{Tr}\left(\Delta_{2 R}^{\dagger} \Delta_{2 R}\right)\right)+\left|\lambda_{R} \operatorname{Tr}\left(\Delta_{1 R} \Delta_{2 R}\right)+\lambda_{S} S^{2}\right|^{2} \\
& +y_{4}^{L} \lambda_{R}\left(S^{*} \tilde{L}_{R}^{T} \Delta_{2 R}^{\dagger} \epsilon \tilde{L}_{R}+\text { h.c. }\right)+\left(y_{4}^{L}\right)^{2}\left(\tilde{L}_{R}^{\dagger} \tilde{L}_{R}\right)^{2} \\
V_{D}= & \frac{g_{R}^{2}}{2} \sum_{i=1}^{3}\left(\operatorname{Tr}\left(\Delta_{1 R}^{\dagger}\left[\tau_{i}, \Delta_{1 R}\right]\right)+\operatorname{Tr}\left(\Delta_{2 R}^{\dagger}\left[\tau_{i}, \Delta_{2 R}\right]\right)-\tilde{L}_{R}^{\dagger} \tau_{i} \tilde{L}_{R}\right)^{2} \\
& +\frac{g_{B L}^{2}}{2}\left(\operatorname{Tr}\left(\Delta_{2 R}^{\dagger} \Delta_{2 R}\right)-\operatorname{Tr}\left(\Delta_{1 R}^{\dagger} \Delta_{1 R}\right)+\frac{1}{2} \tilde{L}_{R}^{\dagger} \tilde{L}_{R}\right) \\
V_{\text {soft }}= & m_{\Delta_{1 R}}^{2} \operatorname{Tr}\left(\Delta_{1 R} \Delta_{1 R}^{\dagger}\right)+m_{\Delta_{2 R}}^{2} \operatorname{Tr}\left(\Delta_{2 R} \Delta_{2 R}^{\dagger}\right)+m_{S}^{2}|S|^{2}+m_{L_{R}}^{2} \tilde{L}_{R}^{\dagger} \tilde{L}_{R} \\
& +\left(T_{\lambda_{R}} S \operatorname{Tr}\left(\Delta_{1 R} \Delta_{2 R}\right)+\frac{1}{3} T_{\lambda_{S}} S^{3}+T_{4}^{L} \tilde{L}_{R}^{T} \Delta_{1 R} \epsilon \tilde{L}_{R}+\text { h.c. }\right) .
\end{aligned}
$$

In these expressions, $\tau_{i}=\frac{1}{2} \sigma_{i}$, where $\sigma_{i}$ are the Pauli matrices. For simplicity we set the trilinear soft supersymmetry-breaking couplings to zero as they do not change the qualitative features that we are aiming to describe.

We start by removing the soft supersymmetry-breaking masses $m_{\Delta_{1 R}}^{2}, m_{\Delta_{2 R}}^{2}$ and $m_{S}^{2}$ from the equations by requiring that the tadpole equations for the desired charge-conserving case of eq. (2.2) are solved,

$$
\left.\frac{\partial V_{0}}{\partial X}\right|_{\mathrm{DSB}:\left\langle\Delta_{i R}^{0}\right\rangle=\frac{v_{i R}}{\sqrt{2}},\langle S\rangle=\frac{v_{S}}{\sqrt{2}}}=0 \quad \text { for } \quad X=\left\{\Delta_{1 R}^{0}, \Delta_{2 R}^{0}, S\right\},
$$

so that the expression of $V_{\text {soft }}$ is optimized for the DSB case. However, as argued above, the global minimum of $V_{0}$ consists of a configuration where the vevs of the triplet fields are aligned along the $\tau_{1}$ direction. Taking the effect of the soft supersymmetry-breaking masses into account, the magnitudes of $v_{i R}$ (with $i=1,2$ ) get slightly modified by factors $\alpha_{i}$ which are close to one, so that we can relate the vevs derived in the DSB case to those derived in the $\mathrm{CB}$ case,

$$
\left.\left\langle\Delta_{1 R}^{0}\right\rangle\right|_{\mathrm{CB}}=\left.\left\langle\Delta_{1 R}^{--}\right\rangle\right|_{\mathrm{CB}}=\alpha_{1} \frac{v_{1 R}}{2} \quad \text { and }\left.\quad\left\langle\Delta_{2 R}^{0}\right\rangle\right|_{\mathrm{CB}}=\left.\left\langle\Delta_{2 R}^{++}\right\rangle\right|_{\mathrm{CB}}=\alpha_{2} \frac{v_{2 R}}{2} .
$$

Consequently, we trade the tadpole equations for the doubly-charged fields with

$$
\frac{\left.\partial V_{0}\right|_{\mathrm{CB}}}{\partial \alpha_{i}}=0
$$

For the evaluation of the mass spectrum at the DSB and the CB minima, we split all complex scalar fields into their scalar and pseudoscalar components as for the CB minimum, as electromagnetism is eventually broken. Taking into account all possible vevs, we rewrite every scalar field $X$ as $X=\frac{1}{\sqrt{2}}\left(v_{X}+\phi_{X}^{S}+i \phi_{X}^{P}\right)$. After rotating out the unphysical would-be Goldstone bosons in each configuration, we compute a $15 \times 15$ scalar mass matrix

$$
\left(M_{S}^{2}\right)_{i j}^{\mathrm{DSB} / \mathrm{CB}}=\left.\frac{\partial^{2} V_{0}}{\partial \phi_{i} \partial \phi_{j}}\right|_{\mathrm{DSB} / \mathrm{CB}} .
$$


The fermionic part of the spectrum can be evaluated for the $\mathrm{SU}(2)_{R}\left(\mathrm{U}(1)_{B-L}\right)$ gauginos $\tilde{W}_{R}^{i}(\tilde{B})$, Higgsino triplets $\tilde{\Delta}_{i R}$ as well as the the singlet fermion $\tilde{S}$ and the lepton doublet $L_{R}$ from the Lagrangian terms

$$
\begin{aligned}
\mathcal{L}_{\text {mass }}^{\text {fermions }}=- & \lambda_{S} S \tilde{S} \tilde{S}-\lambda_{R}\left(\tilde{S} \operatorname{Tr}\left(\tilde{\Delta}_{1 R} \Delta_{2 R}\right)+\tilde{S} \operatorname{Tr}\left(\Delta_{1 R} \tilde{\Delta}_{2 R}\right)+S \operatorname{Tr}\left(\tilde{\Delta}_{1 R} \tilde{\Delta}_{2 R}\right)\right) \\
& -\frac{y_{4}^{L}}{2}\left(2 L_{R}^{T} \Delta_{1 R} \epsilon L_{R}+\tilde{L}_{R}^{T} \tilde{\Delta}_{1 R} \epsilon L_{R}+L_{R}^{T} \tilde{\Delta}_{1 R} \epsilon \tilde{L}_{R}\right) \\
& -\sqrt{2} g_{R} \sum_{i=1}^{3} \tilde{W}_{R, i}\left(\operatorname{Tr}\left(\Delta_{1 R}^{\dagger}\left[\tau_{i}, \tilde{\Delta}_{1 R}\right]\right)+\operatorname{Tr}\left(\Delta_{2 R}^{\dagger}\left[\tau_{i}, \tilde{\Delta}_{2 R}\right]\right)-\tilde{L}_{R}^{\dagger} \tau_{i} L_{R}\right) \\
& -\sqrt{2} g_{B L} \tilde{B}\left(\operatorname{Tr}\left(\Delta_{2 R}^{\dagger} \tilde{\Delta}_{2 R}\right)-\operatorname{Tr}\left(\Delta_{1 R}^{\dagger} \tilde{\Delta}_{1 R}\right)+\frac{1}{2} \tilde{L}_{R}^{\dagger} \tau_{i} L_{R}\right) \\
& -\frac{1}{2} m_{\tilde{W}_{R}} \sum_{i=1}^{3} \tilde{W}_{R, i} \tilde{W}_{R, i}-\frac{1}{2} m_{\tilde{B}} \tilde{B} \tilde{B}+\text { h.c. }
\end{aligned}
$$

As an example for the differences between both vacuum configurations, the lepton masses are given by

$$
\begin{aligned}
\left.m_{\nu_{R}}\right|_{\mathrm{DSB}} & =\sqrt{2} v_{1 R} y_{4}^{L}, & \left.m_{e_{R}}\right|_{\mathrm{DSB}} & =0 \\
\left.m_{\nu_{R}}\right|_{\mathrm{CB}} & =\alpha_{1} v_{1 R} y_{4}^{L}, & \left.m_{e_{R}}\right|_{\mathrm{CB}} & =\alpha_{1} v_{1 R} y_{4}^{L} .
\end{aligned}
$$

Finally, the masses of the vector bosons are derived from the non-derivative part of the gauge-invariant kinetic terms of the Higgs bosons,

$$
\begin{aligned}
\mathcal{L}_{\text {mass }}^{\text {vector }}= & \operatorname{Tr}\left(\left(g_{R} \sum_{a=1}^{3} W_{R}^{\mu, a}\left[\Delta_{1 R}^{\dagger}, \tau^{a}\right]-g_{B L} B^{\mu} \Delta_{1 R}^{\dagger}\right)\left(g_{R} \sum_{b=1}^{3} W_{R, \mu}^{b}\left[\tau^{b}, \Delta_{1 R}\right]-g_{B L} B_{\mu} \Delta_{1 R}\right)\right. \\
& \left.+\left(g_{R} \sum_{a=1}^{3} W_{R}^{\mu, a}\left[\Delta_{2 R}^{\dagger}, \tau^{a}\right]+g_{B L} B^{\mu} \Delta_{2 R}^{\dagger}\right)\left(g_{R} \sum_{b=1}^{3} W_{R, \mu}^{b}\left[\tau^{b}, \Delta_{2 R}\right]+g_{B L} B_{\mu} \Delta_{2 R}\right)\right) .
\end{aligned}
$$

This gives three heavy states of masses of $\mathcal{O}\left(v_{R}\right)$ and one massless state for each vacuum structure. In the DSB case, the hypercharge symmetry group remains unbroken by the triplet vevs and the associated boson is thus massless. In the CB case, the vevs are aligned along the $\tau_{1}$ direction so that this generator remains unbroken $\left(\left[\tau_{1}, \Delta_{i R}\right]=0\right)$ and the $W_{R, 1}$ boson turns out to be massless. This is a consequence of the fact that a Higgs field in the adjoint representation of an $\mathrm{SU}(N)$ group cannot break the rank of this group.

We now move on with the analysis of the one-loop effective potential

$$
V_{1 L, \text { eff. }}=V_{0}+V_{\mathrm{CW}}
$$

where $V_{\mathrm{CW}}$ is the Coleman-Weinberg potential. It reads, in the $\overline{\mathrm{DR}}$ scheme and using Landau gauge,

$$
V_{\mathrm{CW}}=\sum_{n} \frac{(-1)^{2 s_{n}}\left(2 s_{n}+1\right)}{64 \pi^{2}} m_{n}^{4}\left(\log \left(\frac{m_{n}^{2}}{Q^{2}}\right)-\frac{3}{2}\right)
$$



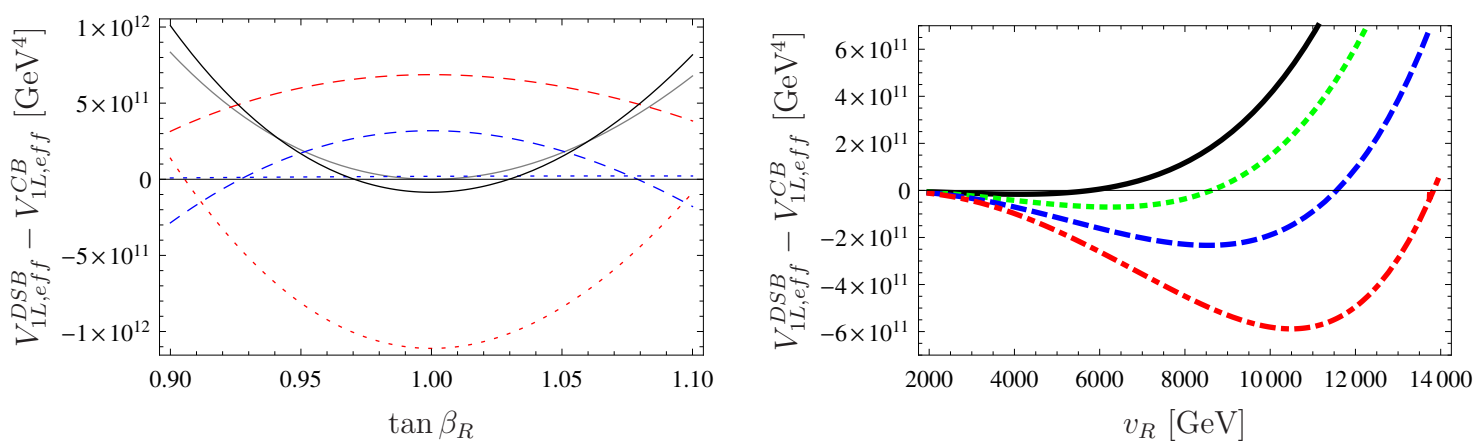

Figure 10. Differences of the scalar potential depths between the DSB and the CB cases at the one-loop level. For negative (positive) values of this difference, the DSB (CB) minimum is the global one. In the left panel of the figure, we represent this difference at the tree (solid grey line) and at the one-loop (solid black line) level as a function of $\tan \beta_{R}$ for $v_{R}=5.5 \mathrm{TeV}, \lambda_{R}=0.4$, $y_{4}^{L}=0.25, m_{L_{R}}^{2}=2 \cdot 10^{6} \mathrm{GeV}^{2}$ and $v_{S}=10 \mathrm{TeV}$. In addition we split the results in terms of the various contributions: slepton and lepton fields (blue dotted line), Higgs fields (blue dashed line), gauginos/Higgsinos (red dotted line) and vector bosons (red dashed line). In the right panel of the figure, we depict the total difference for $\tan \beta_{R}=1.02, y_{4}^{L}=0$ and $\lambda_{R}=0.3$ (solid black line), 0.4 (green dotted line), 0.5 (blue dashed line) and 0.6 (red dot-dashed line).

where $n$ runs over all real scalar fields, Weyl fermions and gauge bosons. We denote by $s_{n}$ and $m_{n}$ the respective spin and mass of the $n^{\text {th }}$ field and have also set the renormalization scale $Q$ to $1 \mathrm{TeV}$. We show on the left panel of figure 10 the difference

$$
\Delta V=V_{1 L, \text { eff. }}^{\mathrm{DSB}}-V_{1 L, \text { eff. }}^{\mathrm{CB}}
$$

at the tree-level, at the one-loop level as well as after breaking down the result for the different contributions to the potential. For the chosen set of parameters, the global minimum is of the DSB kind up for $\tan \beta_{R}$ values in the [0.97, 1.03] range. As illustrated on this figure, the observed behaviour is a consequence of the interplay between the fermionic and the bosonic contributions in the Higgs and gauge sectors. In particular, the DSB vacuum can be the global minimum only due to the fermionic contributions. This may seem to contradict the results of the previous section where the charged Higgs and $W_{R}$ diagrams are very important for getting a non-tachyonic doubly-charged Higgs. We however recall that we are focusing here on the differences between the minima and not on the absolute contributions.

We have shown in section 3 that a sizeable $\lambda_{R}$ is needed to get $m_{H^{ \pm \pm}}^{2}$ positive. On the right panel of figure 10, we study the dependence of $\Delta V$ on $v_{R}$ for different values of $\lambda_{R}$. We observe that increasing $\lambda_{R}$ also increases the relative depth of the DSB vacuum with respect to the charge-breaking one. We have finally checked that the features discussed so far do not depend on the choice of $Q$ by varying it up to $2 v_{R}$ and evolving the model parameters according to the renormalization group equations.

We now turn to the study of the full model and allow for additional vevs. We have used SARAH to produce a Vevacious code [48] for the left-right supersymmetric models under study. The Vevacious programme starts by evaluating the tree-level scalar potential for 
a given spectrum and finds all extrema in terms of all scalar particle vevs, given together with the corresponding value of the potential. In a second step, the nearby extrema are found and evaluated using a full one-loop potential. In principle one should allow for all scalars to obtain a vev which however in models like the one considered in this work would take an enormous amount of CPU time well beyond a year for a single point of parameter space. Therefore, we restrict ourselves to vacuum configurations in which only the $\Phi_{1,2}^{0}$, $\Delta_{1 R, 2 R}^{0}$ and $S$ neutral fields and the $\Delta_{1 R}^{--}$and $\Delta_{2 R}^{++}$doubly-charged fields could develop non-vanishing vacuum expectation values. We further allow for one generation of right sneutrinos to receive a vev in order to cover the possibility of a charge-conserving but $R$-parity violating vacuum.

Under these assumptions, in the parameter regions where an $R$-parity conserving spectrum can be found at the one-loop level, the global minimum is found to be always either of the desired kind of eq. (2.2), such that

$$
\begin{aligned}
& \left\langle\Phi_{1}^{0}\right\rangle=v_{d} / \sqrt{2}, \quad\left\langle\Phi_{2}^{0}\right\rangle=v_{u} / \sqrt{2}, \quad\langle S\rangle=v_{S} / \sqrt{2}, \quad\left\langle\tilde{\nu}^{c}\right\rangle=0, \\
& \left\langle\Delta_{1 R}^{0}\right\rangle=v_{1 R} / \sqrt{2}, \quad\left\langle\Delta_{1 R}^{--}\right\rangle=0, \quad\left\langle\Delta_{2 R}^{0}\right\rangle=v_{2 R} / \sqrt{2}, \quad\left\langle\Delta_{2 R}^{++}\right\rangle=0,
\end{aligned}
$$

or the charge-breaking nature of eq. (2.3) with

$$
\begin{aligned}
\left\langle\Phi_{1}^{0}\right\rangle & =\left\langle\Phi_{2}^{0}\right\rangle=0, & \langle S\rangle & =v_{S} / \sqrt{2}, \quad\left\langle\tilde{\nu}^{c}\right\rangle=0, \\
\left\langle\Delta_{1 R}^{0}\right\rangle & =\left\langle\Delta_{1 R}^{--}\right\rangle \simeq v_{1 R} / 2, & \left\langle\Delta_{2 R}^{0}\right\rangle & =\left\langle\Delta_{2 R}^{++}\right\rangle \simeq v_{2 R} / 2 .
\end{aligned}
$$

In figure 11 , we depict in the $\left(v_{R}, y_{4}^{L}\right)$ plane the regions of the parameter space where the desired vacuum configuration corresponds to the global minimum of the scalar potential (green) and the ones where the charge-breaking configuration is preferred (red). We have found that regions relevant for LHC physics (i.e., regions where the mass of the doublycharged Higgs is above the current limits) are not only exhibiting a viable local minimum but also a global viable one. Moreover, as for the $y_{4}^{L}$-dependent loop corrections to the $H^{ \pm \pm}$ mass, larger the $y_{4}^{L}$ and $v_{R}$ values are, the less favoured is the benchmark scenario under consideration. Furthermore, as illustrated in the right panel of the figure, a larger value of $\lambda_{R} v_{S}$ has the virtue of pulling the desired vacuum configuration into a deeper minimum. However, as already above-mentioned, this increases the possibility of a tachyonic right sneutrino due to large negative $F$-term contributions proportional to the $\lambda_{R} v_{S}$ product. This happens at the tree level in the parameter space above the black line. In the region indicated by light grey shading we consequently find $R$-parity violating global minima, i.e. $\left\langle\tilde{\nu}^{c}\right\rangle \neq 0$, which still conserve electric charge but feature too large electroweak vevs of the order of one $\mathrm{TeV}$. In the dark grey area we find minima which break both, $R$-parity and electric charge. We stress that this $R$-parity violation is solely due to the negative $F$-term contribution which drives a sneutrino tachyonic and could be evaded by a larger soft slepton mass. It is therefore of completely different origin than the $R$-parity violation suggested in early studies of this kind of models $[12,37]$. Finally, considering more than one right-handed neutrino leads to a reduction of both the favoured region and the mass of the doubly-charged Higgs boson. The blue lines in figure 11 show the separation between the red and the green area as evaluated from the simplified model discussed in the beginning of 

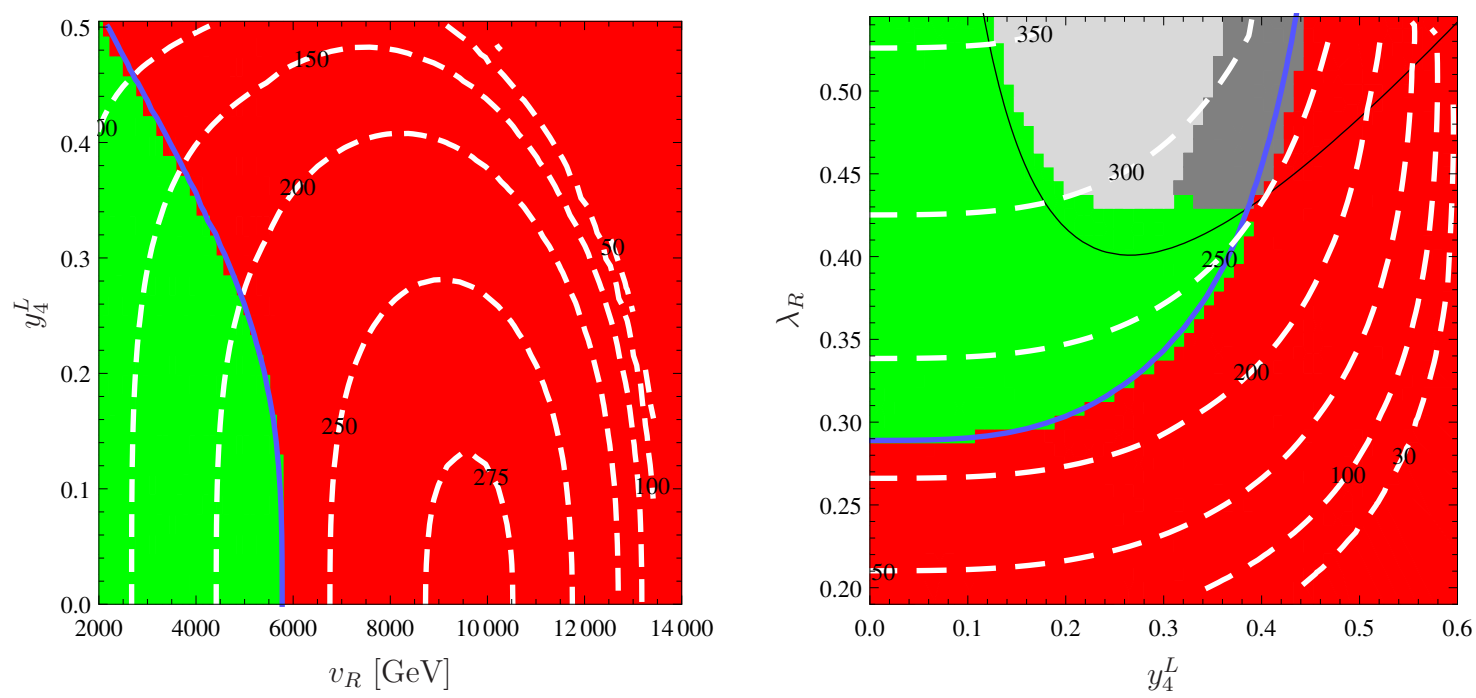

Figure 11. Analysis of the vacuum stability. The results are presented in the $\left(v_{R}, y_{4}^{L}\right)$ plane with $\lambda_{R}=0.3$ and for one generation of right-handed neutrinos (left) and in the $\left(y_{4}^{L}, \lambda_{R}\right)$ plane with $v_{R}=5.5 \mathrm{TeV}$ (right). The other model parameters have been fixed to $\tan \beta_{R}=1.02, m_{L_{R}}^{2}=$ $2 \cdot 10^{6} \mathrm{GeV}^{2}$ and $v_{S}=10 \mathrm{TeV}$. The white contours depicted on the figures indicate isomass lines for the doubly-charged Higgs boson in $\mathrm{GeV}$ as obtained with our full one-loop-corrected calculation. The green regions correspond to setups where the vacuum configuration of eq. (2.2) is the global minimum of the scalar potential whereas in the red regions, the charge-breaking vacuum configuration consists of a deeper minimum. The blue lines separate the cases of a deeper DSB minimum and a deeper CB minimum in the context of the simplified model discussed above. The parameter space above the black line features a tachyonic sneutrino at the tree level and scenarios in the grey regions consequently violate $R$-parity while conserving (lighter grey) or breaking (darker grey) electric charge.

this section. This simplified description can thus safely be used to get rough information on which of the two minima is the deeper one. It does however not cover the case of $R$-parity violating global minima as one would need to extend the simplified setup with vevs for the sneutrino fields.

Scenarios for which the global minimum is charge-breaking could nevertheless be viable in cases where the tunneling time from the local $\mathrm{SU}(2)_{L} \times \mathrm{U}(1)_{Y}$ minimum to the global one is sufficiently large. The decay of a vacuum state into a deeper minimum can be described by the phase transition in which bubbles of true vacuum nucleate out of a false vacuum state. For a successful phase transition, the bubble has to be of a critical size and can, at zero temperature, be found by minimizing the Euclidean action [49]

$$
S_{E}=\int \mathrm{d}^{4} x\left(\frac{1}{2}\left(\partial_{\mu} \vec{\phi}\right)\left(\partial^{\mu} \vec{\phi}\right)+V(\vec{\phi})\right)
$$

where $\vec{\phi}$ is a vector of all scalar fields and the coordinate $x^{0}$ corresponds to the imaginary time coordinate $\tau=i t$. Equivalently, the equations of motion have to be solved so that $\delta S_{E}=0$, which eventually determines the optimal tunneling path in field space. For a first estimation of $S_{E}$, the straight tunneling path between two vacua can be used, which 
corresponds to a reduction of the problem to a one-dimensional problem. The latter can be solved numerically to arbitrary precision by the so-called overshoot/undershoot method (see, e.g., ref. [50]). The full path deformation in all field dimensions is computationally extremely expensive and is hardly applicable to the dimensionality of the model studied in this work. The decay rate of the false vacuum per unit volume $\mathcal{V}$ is then given by [49]

$$
\Gamma / \mathcal{V}=A e^{-S_{E}}
$$

In this expression, $A$ is a quantity whose dimension is in the fourth power of energy $E^{4}$ and is related to the eigenvalues of a functional determinant [51]. This determinant is in practice usually estimated on dimensional grounds [50], $A \sim M^{4}$ with $M$ being the typical mass scale of the model.

A first estimate using the direct tunneling path at zero temperature shows that all scenarios included in the excluded region of the figure (red) are metastable which is due to the large separation in field space of the different vacua.

It is however well known from the MSSM $[52,53]$ that allowing for additional vevs and/or including thermal effects can imply that metastable vacua are in fact unstable. A thorough investigation of this effect lies beyond the scope of this paper.

Combining the results of the right panel of figure 11 with the LHC constraint of $v_{R} \gtrsim 4.9 \mathrm{TeV}$ (see section 4 ) implies that all but a small strip of the parameter space turns out to be excluded if one assumes that the right-handed neutrino is of a third generation nature. In contrast, scenarios featuring a right-handed neutrino of one of the first two generations turn out to be excluded. In this way, we have demonstrated how accounting both for theoretical and experimental constraints has allowed us to almost exclude all possible setups in the left-right supersymmetric models under consideration.

\section{Conclusion}

We have studied a specific class of supersymmetric models exhibiting a left-right gauge symmetry and investigated to which extent experimental and theoretical constraints restrict the viable regions of the parameter space. A particular property of this class of models that feature Higgs fields lying in the triplet representation of the $\mathrm{SU}(2)_{R}$ group is that one of the corresponding doubly-charged Higgs bosons gets tachyonic when the scalar potential is minimized at the tree level. Even though it was known that loop corrections taking into account the Yukawa couplings of the right-handed neutrinos to the $\mathrm{SU}(2)_{R}$ Higgs bosons were modifying these conclusions, a complete one-loop calculation was still missing. We have filled this gap and shown that in a large part of the parameter space, the complete one-loop calculation is necessary for reliable predictions.

We have then studied to which extent the phenomenologically viable regions of the parameter space (where the doubly-charged Higgs boson is non-tachyonic), are constrained by experimental data and theoretical considerations concerning the global minimum of the one-loop-corrected scalar potential. We have found that the latter favours lower values of $v_{R}$ whereas the LHC gives tight lower bounds on this vev, in particular by exploring the $W_{R}$ decays into jets. Moreover, the lightest doubly-charged Higgs boson is constrained to 
be well below $1 \mathrm{TeV}$ for Yukawa couplings imposed to lie in the perturbative regime. The left-right supersymmetric setup that we have investigated is therefore close to exclusion, in particular if the future LHC searches for a $W_{R}$ and a doubly-charged Higgs boson do not exhibit any signal within the next few years.

\section{Acknowledgments}

We thank Florian Staub and Ben O'Leary for useful discussions on SARAH and Vevacious as well as Ayon Patra for clarifications on their paper and Adam Alloul for discussions in the early days of this project. M.E.K. and W.P. are supported by the DFG research training group GRK1147 and by the DFG project no. PO-1337/3-1, L.B. and B.F. by the French ANR 12 JS05 00201 BATS@LHC and by the Theory-LHC-France initiative of the CNRS/IN2P3 and Inphynity challenge of the CNRS/INP. W.P. also thanks the CERN theory group, where part of this work has been carried out, for hospitality.

Open Access. This article is distributed under the terms of the Creative Commons Attribution License (CC-BY 4.0), which permits any use, distribution and reproduction in any medium, provided the original author(s) and source are credited.

\section{References}

[1] ATLAS collaboration, Combined search for the Standard Model Higgs boson using up to $4.9 \mathrm{fb}^{-1}$ of pp collision data at $\sqrt{s}=7 \mathrm{TeV}$ with the ATLAS detector at the LHC, Phys. Lett. B 710 (2012) 49 [arXiv:1202.1408] [INSPIRE].

[2] CMS collaboration, Combined results of searches for the standard model Higgs boson in pp collisions at $\sqrt{s}=7 \mathrm{TeV}$, Phys. Lett. B 710 (2012) 26 [arXiv:1202.1488] [INSPIRE].

[3] H.P. Nilles, Supersymmetry, Supergravity and Particle Physics, Phys. Rept. 110 (1984) 1 [INSPIRE].

[4] H.E. Haber and G.L. Kane, The Search for Supersymmetry: Probing Physics Beyond the Standard Model, Phys. Rept. 117 (1985) 75 [INSPIRE].

[5] R.M. Francis, M. Frank and C.S. Kalman, Anomalous magnetic moment of the muon arising from the extensions of the supersymmetric standard model based on left-right symmetry, Phys. Rev. D 43 (1991) 2369 [INSPIRE].

[6] K. Huitu, J. Maalampi and M. Raidal, Slepton pair production in $e^{+} e^{-}$collision in supersymmetric left-right model, Phys. Lett. B 328 (1994) 60 [hep-ph/9402219] [INSPIRE].

[7] K. Huitu, J. Maalampi and M. Raidal, Supersymmetric left-right model and its tests in linear colliders, Nucl. Phys. B 420 (1994) 449 [hep-ph/9312235] [INSPIRE].

[8] K.S. Babu and R.N. Mohapatra, Minimal Supersymmetric Left-Right Model, Phys. Lett. B 668 (2008) 404 [arXiv:0807.0481] [INSPIRE].

[9] K.S. Babu, X.-G. He and E. Ma, New Supersymmetric Left-Right Gauge Model: Higgs Boson Structure and Neutral Current Analysis, Phys. Rev. D 36 (1987) 878 [INSPIRE].

[10] Y. Zhang, H. An, X.-d. Ji and R.N. Mohapatra, Light Higgs Mass Bound in SUSY Left-Right Models, Phys. Rev. D 78 (2008) 011302 [arXiv:0804.0268] [INSPIRE]. 
[11] M.E. Krauss, W. Porod and F. Staub, $\mathrm{SO}(10)$ inspired gauge-mediated supersymmetry breaking, Phys. Rev. D 88 (2013) 015014 [arXiv: 1304.0769] [INSPIRE].

[12] R. Kuchimanchi and R.N. Mohapatra, No parity violation without R-parity violation, Phys. Rev. D 48 (1993) 4352 [hep-ph/9306290] [INSPIRE].

[13] R. Kuchimanchi and R.N. Mohapatra, Upper bound on the $W(R)$ mass in automatically $R$ conserving SUSY models, Phys. Rev. Lett. 75 (1995) 3989 [hep-ph/9509256] [INSPIRE].

[14] Z. Chacko and R.N. Mohapatra, Supersymmetric left-right model and light doubly charged Higgs bosons and Higgsinos, Phys. Rev. D 58 (1998) 015003 [hep-ph/9712359] [INSPIRE].

[15] R.N. Mohapatra and G. Senjanović, Neutrino Mass and Spontaneous Parity Violation, Phys. Rev. Lett. 44 (1980) 912 [INSPIRE].

[16] twiki.cern.ch/AtlasPublic/SupersymmetryPublicResults.

[17] twiki.cern.ch/CMSPublic/PhysicsResultsSUS.

[18] twiki.cern.ch/AtlasPublic/ExoticsPublicResults.

[19] twiki.cern.ch/CMSPublic/PhysicsResultsEXO.

[20] K.S. Babu and A. Patra, Higgs Boson Spectra in Supersymmetric Left-Right Models, arXiv: 1412.8714 [INSPIRE].

[21] CMS collaboration, A search for a doubly-charged Higgs boson in pp collisions at $\sqrt{s}=7 \mathrm{TeV}$, Eur. Phys. J. C 72 (2012) 2189 [arXiv:1207.2666] [INSPIRE].

[22] A. Alloul, M. Frank, B. Fuks and M. Rausch de Traubenberg, Chargino and neutralino production at the Large Hadron Collider in left-right supersymmetric models, JHEP 10 (2013) 033 [arXiv: 1307.5073] [INSPIRE].

[23] G. Brooijmans et al., Les Houches 2013: Physics at TeV Colliders: New Physics Working Group Report, arXiv:1405.1617 [INSPIRE].

[24] F. Staub, SARAH, arXiv:0806.0538 [INSPIRE].

[25] F. Staub, From Superpotential to Model Files for FeynArts and CalcHep/CompHEP, Comput. Phys. Commun. 181 (2010) 1077 [arXiv:0909.2863] [INSPIRE].

[26] F. Staub, Automatic Calculation of supersymmetric Renormalization Group Equations and Self Energies, Comput. Phys. Commun. 182 (2011) 808 [arXiv:1002.0840] [InSPIRE].

[27] F. Staub, SARAH 3.2: Dirac Gauginos, UFO output and more, Comput. Phys. Commun. 184 (2013) pp. 1792-1809 [arXiv: 1207.0906] [INSPIRE].

[28] F. Staub, SARAH 4: A tool for (not only SUSY) model builders, Comput. Phys. Commun. 185 (2014) 1773 [arXiv: 1309.7223] [INSPIRE].

[29] W. Porod, SPheno, a program for calculating supersymmetric spectra, SUSY particle decays and SUSY particle production at $e^{+} e^{-}$colliders, Comput. Phys. Commun. 153 (2003) 275 [hep-ph/0301101] [INSPIRE].

[30] W. Porod and F. Staub, SPheno 3.1: Extensions including flavour, CP-phases and models beyond the MSSM, Comput. Phys. Commun. 183 (2012) 2458 [arXiv:1104.1573] [INSPIRE].

[31] C. Duhr and B. Fuks, A superspace module for the FeynRules package, Comput. Phys. Commun. 182 (2011) 2404 [arXiv:1102.4191] [INSPIRE]. 
[32] A. Alloul, N.D. Christensen, C. Degrande, C. Duhr and B. Fuks, FeynRules 2.0 - A complete toolbox for tree-level phenomenology, Comput. Phys. Commun. 185 (2014) 2250 [arXiv:1310.1921] [INSPIRE].

[33] F. Staub, T. Ohl, W. Porod and C. Speckner, A Tool Box for Implementing Supersymmetric Models, Comput. Phys. Commun. 183 (2012) 2165 [arXiv:1109.5147] [INSPIRE].

[34] F. Staub, Exploring new models in all detail with SARAH, arXiv:1503.04200 [INSPIRE].

[35] J. Alwall et al., The automated computation of tree-level and next-to-leading order differential cross sections and their matching to parton shower simulations, JHEP 07 (2014) 079 [arXiv: 1405.0301] [INSPIRE].

[36] C. Degrande, C. Duhr, B. Fuks, D. Grellscheid, O. Mattelaer and T. Reiter, UFO - The Universal FeynRules Output, Comput. Phys. Commun. 183 (2012) 1201 [arXiv:1108.2040] [INSPIRE].

[37] K. Huitu and J. Maalampi, The Higgs sector of a supersymmetric left-right model, Phys. Lett. B 344 (1995) 217 [hep-ph/9410342] [INSPIRE].

[38] M. Cvetič and J.C. Pati, $N=1$ Supergravity Within the Minimal Left-right Symmetric Model, Phys. Lett. B 135 (1984) 57 [InSPIRE].

[39] ATLAS collaboration, Search for anomalous production of prompt same-sign lepton pairs and pair-produced doubly charged Higgs bosons with $\sqrt{s}=8 \mathrm{TeV}$ pp collisions using the ATLAS detector, JHEP 03 (2015) 041 [arXiv:1412.0237] [INSPIRE].

[40] CMS collaboration, Search for resonances and quantum black holes using dijet mass spectra in proton-proton collisions at $\sqrt{s}=8 \mathrm{TeV}$, Phys. Rev. D 91 (2015) 052009 [arXiv: 1501.04198] [INSPIRE].

[41] CMS collaboration, Search for $W^{\prime} \rightarrow$ tb decays in the lepton + jets final state in $p p$ collisions at $\sqrt{s}=8 \mathrm{TeV}$, JHEP 05 (2014) 108 [arXiv:1402.2176] [INSPIRE].

[42] J. Pumplin, D.R. Stump, J. Huston, H.L. Lai, P.M. Nadolsky and W.K. Tung, New generation of parton distributions with uncertainties from global QCD analysis, JHEP 07 (2002) 012 [hep-ph/0201195] [INSPIRE].

[43] CMS collaboration, Search for heavy neutrinos and $\mathrm{W}$ bosons with right-handed couplings in proton-proton collisions at $\sqrt{s}=8 \mathrm{TeV}$, Eur. Phys. J. C 74 (2014) 3149 [arXiv:1407.3683] [INSPIRE].

[44] S. Bar-Shalom, G. Eilam, T. Han and A. Soni, Charged Higgs Boson Effects in the Production and Decay of a Heavy Majorana Neutrino at the CERN LHC, Phys. Rev. D 77 (2008) 115019 [arXiv:0803.2835] [INSPIRE].

[45] F.F. Deppisch, T.E. Gonzalo, S. Patra, N. Sahu and U. Sarkar, Signal of Right-Handed Charged Gauge Bosons at the LHC?, Phys. Rev. D 90 (2014) 053014 [arXiv:1407.5384] [INSPIRE].

[46] M. Heikinheimo, M. Raidal and C. Spethmann, Testing Right-Handed Currents at the LHC, Eur. Phys. J. C 74 (2014) 3107 [arXiv:1407.6908] [inSPIRE].

[47] S.R. Coleman and E.J. Weinberg, Radiative Corrections as the Origin of Spontaneous Symmetry Breaking, Phys. Rev. D 7 (1973) 1888 [INSPIRE].

[48] J.E. Camargo-Molina, B. O'Leary, W. Porod and F. Staub, Vevacious: A Tool For Finding The Global Minima Of One-Loop Effective Potentials With Many Scalars, Eur. Phys. J. C 73 (2013) 2588 [arXiv:1307.1477] [INSPIRE]. 
[49] S.R. Coleman, The Fate of the False Vacuum. 1. Semiclassical Theory, Phys. Rev. D 15 (1977) 2929 [Erratum ibid. D 16 (1977) 1248] [INSPIRE].

[50] C.L. Wainwright, CosmoTransitions: Computing Cosmological Phase Transition Temperatures and Bubble Profiles with Multiple Fields, Comput. Phys. Commun. 183 (2012) 2006 [arXiv: 1109.4189] [INSPIRE].

[51] C.G. Callan Jr. and S.R. Coleman, The Fate of the False Vacuum. 2. First Quantum Corrections, Phys. Rev. D 16 (1977) 1762 [InSPIRE].

[52] J.E. Camargo-Molina, B. O'Leary, W. Porod and F. Staub, Stability of the CMSSM against sfermion VEVs, JHEP 12 (2013) 103 [arXiv:1309.7212] [INSPIRE].

[53] J.E. Camargo-Molina, B. Garbrecht, B. O'Leary, W. Porod and F. Staub, Constraining the Natural MSSM through tunneling to color-breaking vacua at zero and non-zero temperature, Phys. Lett. B 737 (2014) 156 [arXiv: 1405.7376] [InSPIRE]. 